\title{
\begin{tabular}{l|l} 
Mibraries & DSpace@MIT
\end{tabular}
}

\author{
MIT Open Access Articles
}

\section{Analytic energy gradients for constrained DFT-configuration interaction}

The MIT Faculty has made this article openly available. Please share how this access benefits you. Your story matters.

Citation: Kaduk, Benjamin, Takashi Tsuchimochi, and Troy Van Voorhis. "Analytic Energy Gradients for Constrained DFT-Configuration Interaction." The Journal of Chemical Physics 140, no. 18 (May 14, 2014): 18A503.

As Published: http://dx.doi.org/10.1063/1.4862497

Publisher: American Institute of Physics (AIP)

Persistent URL: http://hdl.handle.net/1721.1/96256

Version: Author's final manuscript: final author's manuscript post peer review, without publisher's formatting or copy editing

Terms of use: Creative Commons Attribution-Noncommercial-Share Alike 


\title{
Analytic Energy Gradients for Constrained DFT-Configuration Interaction
}

\author{
Benjamin Kaduk, Takashi Tsuchimochi, and Troy Van Voorhis* \\ Massachusetts Institute of Technology \\ 77 Massachusetts Avenue \\ Cambridge, MA 02139
}

\begin{abstract}
The constrained density functional theory-configuration interaction (CDFT-CI) method has previously been used to calculate ground-state energies and barrier heights, and to describe electronic excited states, in particular conical intersections. However, the method has been limited to evaluating the electronic energy at just a single nuclear configuration, with the gradient of the energy being available only via finite difference. In this paper, we present analytic gradients of the CDFTCI energy with respect to nuclear coordinates, which gives the potential for accurate geometry optimization and molecular dynamics on both the ground and excited electronic states, a realm which is currently quite challenging for electronic structure theory. We report the performance of CDFT-CI geometry optimization for representative reaction transition states as well as molecules in an excited state. The overall accuracy of CDFT-CI for computing barrier heights is essentially unchanged whether the energies are evaluated at geometries obtained from QCISD or CDFT-CI, indicating that CDFT-CI produces very good reaction transition states. These results open up tantalizing possibilities for future work on excited states.
\end{abstract}

\footnotetext{
*tvan@mit.edu
} 


\section{INTRODUCTION}

Electronic excited states are of interest in a great many chemical systems, being of relevance to photochemistry,[1-8] photodamage to DNA,[9-18] organic semiconductors,[19-23] and more.[24-26] Of particular interest is the dynamics on the excited state, after an excitation event has occurred. In order to study the geometric relaxation of electronic excited states, one requires the force experienced by the nuclei on the excited-state PES. This requirement limits the spectrum of electronic structure methods which are usable, with the field being limited to time-dependent density functional theory (TD-DFT),[27-35] configurationinteraction singles (CIS), [7, 31, 36-41] complete active space self-consistent field (CASSCF) and its second order perturbation theory (CASPT2)[42-48] (note that analytic gradients for CASPT2 have become available only recently[49]) equation-of-motion coupled-cluster singles and doubles (EOM-CCSD)[50-54] and its approximate form (EOM-CC2),[31, 55-61] and sometimes multi-reference configuration interaction (MRCI).[62-65] Even for EOMCCSD, CASPT2, and MRCI, the computational expense will vary with the implementation and application, and such calculations become impractical for systems with more than 10 or 15 atoms.

On the other hand, DFT methods gain a significant advantage of practicality as the system size increases. TD-DFT has seen broad use for electronic excited states in general, and excited-state dynamics and geometry optimization are no exception.[30-35] However, it still suffers from the deficiencies in describing multiple excitations and charge-transfer excitations which render it a less-than-general solution for vertical excitation energy calculations, [66-75] though recent developments show the state of affairs may be improving.[76-79] Restricted open-shell Kohn-Sham (ROKS) [80-82] is a state-specific method, and its gradients are easily available compared to TD-DFT.[83] However, ROKS consists of a two-determinant wave function, and therefore cannot describe electronic structures with multiple excitations. Constrained DFT (CDFT) is designed to directly construct charge- and spin-constrained states and as such can find charge-transfer states directly, using self-consistent ground state techniques.[84-86] The self-consistent nature of the solution means that nonlinear response of the density is included, and hence in principle permits the treatment of multiple excitations from the ground state. However, CDFT has limitations of its own; it is still a single-reference method (and thus suffers from the limitations of DFT in the face of strong 
static correlation), and it has no effective prescription for describing valence excitations. CDFT-configuration interaction (CDFT-CI) explicitly introduces multiple configurations to the electronic structure treatment and a promolecule correction for constraint values, which greatly improves results for situations where static correlation is strongly present, such as dissociation curves and reaction transition states.[87, 88] Additionally, it can treat the ground state and excited states on the same footing, which is necessary for describing conical intersections qualitatively.[67] However, applications of CDFT-CI have heretofore remained somewhat limited due to the unavailability of gradients of the electronic energy. In this work, we present the theory and implementation of analytic energy gradients for CDFTCI. These forces are used to optimize the transition-state geometries for a standard set of reaction barriers. In most cases, the energy does not change noticeably from the reference transition-state geometry to the optimized geometry, indicating that CDFT-CI-optimized geometries for transition states are of comparable quality to the reference geometries. Using CDFT-CI gradients for geometry optimization on the excited state also converges to geometries of acceptable quality, promising the capability of handling the hard cases of, for example, conical intersections and states with significant charge-transfer and valence mixing.

This paper is organized as follows. In Sec. II A, our general strategy for obtaining the CDFT-CI energy gradients is outlined. Sec. II B shows the derivatives of matrix elements and couplings as well as how to avoid those of molecular orbitals (MO) with respect to nuclear positions. Sec. II C presents the contribution to the gradient of the promolecule correction introduced for CDFT-CI. The constraint potential contribution to the gradient is given in Sec. II D, and Sec. II E summarizes the overall expressions derived. In Sec. III we evaluate the performance of the CDFT-CI gradients with the HTBH38/04 and NHTBH38 sets for benchmark calculations on transition states, and on excited states of small molecules. Finally, we draw our conclusions and perspective on the present study in Sec. IV.

\section{METHODS}

We briefly summarize the equations of CDFT and CDFT-CI before proceeding to the derivation of expressions for the gradient of the energy. CDFT takes as input a density functional giving the energy $E[\rho]$ and adds a constraint Lagrange multiplier term to yield a 
new functional

$$
E\left[\rho, V_{k}\right]=E[\rho]+\sum_{k} V_{k}\left(\int \hat{w}_{k} \rho(\mathbf{r}) d \mathbf{r}-N_{k}\right),
$$

where $\hat{w}_{k}$ is a "weight" operator that probes the number of electrons in some particular region of space and $N_{k}$ is a target value for that operator. Minimizing $E$ with respect to $\rho$ under the constraints imposed yields a state with the desired constrained charge and spin properties. The effect of the constraint terms in the energy expression are equivalent to adding an additional "constraint" potential $\sum_{k} V_{k} \hat{w}_{k}(\mathbf{r})=\sum_{k} \hat{V}_{k}(\mathbf{r})=\hat{V}$ acting on the electrons in Kohn-Sham theory.

CDFT-CI requires the user to specify a collection of different constrained states which are used as a basis/active space for constructing a configuration-interaction matrix; the basis states are specified as integer and half-integer charge and spin constraints on particular fragments of the system in question, which are converted into physically attainable values using the promolecule correction. It should be emphasized that the CDFT basis states for CDFT-CI are completely unrelated to each other: they share no orbitals, and experience different constraint potentials. This is in contrast to the traditional CI methods, where the CI basis states are formed as excitations from one or more reference determinants. The CI matrix of CDFT basis states gives the CDFT-CI eigenvalue equation,

$$
\left(\begin{array}{ll}
\mathcal{H}_{11} & \mathcal{H}_{12} \\
\mathcal{H}_{21} & \mathcal{H}_{22}
\end{array}\right)\left(\begin{array}{l}
C_{1} \\
C_{2}
\end{array}\right)=\mathcal{E}\left(\begin{array}{cc}
\mathcal{S}_{11} & \mathcal{S}_{12} \\
\mathcal{S}_{21} & \mathcal{S}_{22}
\end{array}\right)\left(\begin{array}{l}
C_{1} \\
C_{2}
\end{array}\right)
$$

We show only the two-state case, but the generalization to $N$ states is easily made. The diagonal elements of $\mathbf{H}$ are just the energies of the constrained states that form the basis for the active space; the off-diagonal elements are constructed as:[87, 88]

$$
\mathcal{H}_{I J}=\mathcal{H}_{J I}=\frac{F_{I}+F_{J}}{2} \mathcal{S}_{I J}-\left\langle\Phi_{I}\left|\frac{\hat{V}_{I}+\hat{V}_{J}}{2}\right| \Phi_{J}\right\rangle,
$$

where $\Phi_{I}$ is the Kohn-Sham determinant for the $I$ th CDFT state, $F_{I}$ is the energy of the $I$ th CDFT state in the presence of the constraining potential $\hat{V}_{I}$ for state $I$, and $\mathcal{S}_{I J}$ is just $\left\langle\Phi_{I} \mid \Phi_{J}\right\rangle$. For the rest of this work, we will assume the two-state form, using capital letters $I$ and $J$ to indicate the different states; extension to the $N$ state case is straightforward. We also adhere to the convention that $i$ and $j$ index occupied orbitals, $p$ and $q$ index all orbitals, and Greek letters index atomic orbitals (AO). 
If we write $\mathbf{H C}=\mathcal{E} \mathbf{S C}$, then we can easily take the derivative with respect to a nuclear coordinate $x$ and write

$$
\mathbf{H}^{x} \mathbf{C}+\mathbf{H C}^{x}=\mathcal{E}^{x} \mathbf{S C}+\mathcal{E} \mathbf{S}^{x} \mathbf{C}+\mathcal{E} \mathbf{S C}^{x} .
$$

Bracketing on the left with $\mathbf{C}^{\dagger}$ and rearranging lets us solve for the gradient $\mathcal{E}^{x}$ :

$$
\mathcal{E}^{x}=\mathbf{C}^{\dagger}\left(\mathbf{H}^{x}-\mathcal{E} \mathbf{S}^{x}\right) \mathbf{C}
$$

$\mathcal{E}$ and $\mathbf{C}$ are already known from the single-point energy evaluation, so the only new terms required for the gradient expression are $\mathbf{H}^{x}$ and $\mathbf{S}^{x}$.

\section{A. Overview}

In order to actually use Eq. (5) to obtain $\mathcal{E}^{x}$, we must consider both diagonal terms of the form $\mathbf{H}_{I I}^{x}$ and off-diagonal terms $\mathbf{H}_{I J}^{x}$ and $\mathbf{S}_{I J}^{x}$. (There are no diagonal overlap terms, since normalized states will always have unit self-overlap.) The diagonal terms $\mathbf{H}_{I I}^{x}$ are just the

energy gradient of the constrained states, so we focus on the off-diagonal elements $\mathbf{H}_{I J}^{x}$ and $\mathbf{S}_{I J}^{x}$. However, since we only use these two quantities in combination, it proves convenient to define an auxiliary quantity $\mathbf{W}=\mathbf{H}-\mathcal{E} \mathbf{S}$ where $\mathcal{E}$ is treated as a constant and does not vary with changes in any other parameters. We then seek to compute $\mathbf{W}^{x}=(\mathbf{H}-\mathcal{E} \mathbf{S})^{x}=$ $\mathbf{H}^{x}-\mathcal{E} \mathbf{S}^{x}$.

At this point, the high-level view is no longer sufficient and we must expand the expression to include the Kohn-Sham determinants of our CI basis states.

$$
\begin{aligned}
W_{I I} & =H_{I I}-\mathcal{E} S_{I I} \\
W_{I I}^{x} & =H_{I I}^{x} \\
W_{I J} & =\left(\frac{F_{I}+F_{J}}{2}-\mathcal{E}\right)\left\langle\Phi_{I} \mid \Phi_{J}\right\rangle-\left\langle\Phi_{I}\left|\frac{\hat{V}_{I}+\hat{V}_{J}}{2}\right| \Phi_{J}\right\rangle \\
W_{I J}^{x} & =\frac{F_{I}^{x}+F_{J}^{x}}{2} \mathcal{S}_{I J}-\left\langle\Phi_{I}\left|\frac{\hat{V}_{I}^{x}+\hat{V}_{J}^{x}}{2}\right| \Phi_{J}\right\rangle+\left\langle\Phi_{I}^{x}|\hat{O}| \Phi_{J}\right\rangle+\left\langle\Phi_{I}|\hat{O}| \Phi_{J}^{x}\right\rangle \\
\hat{O} & =\left(\frac{F_{I}+F_{J}}{2}-\mathcal{E}\right)-\frac{\hat{V}_{I}+\hat{V}_{J}}{2}
\end{aligned}
$$

Equations (6) and (8) follow directly from the definition of $\mathbf{W}$ and equation (3). However, the terms in $\left\langle\Phi_{I}^{x}\right|$ and $\left|\Phi_{J}^{x}\right\rangle$ are unreasonable to compute, given that the wavefunction gradient 
requires $O\left(N^{3}\right)$ space to store and $O\left(N^{5}\right)$ time to compute. As such, we seek an alternate route to $W_{I J}^{x}$ which does not involve the gradient of the wavefunction; we will adopt the standard framework of making the matrix element variational.[49]

The procedure to evaluate Eq. (9) does not have a linear logical order of execution; to assist in understanding the steps involved in the computation, a flow chart of the relevant expressions is presented in Figure 1. The labeled boxes in the flowchart correspond roughly to the subsections that follow, though we start off with the general computation of the gradient of a matrix element in Sec IIB, used for both the promolecule contribution (Sec II C), which is required to carry out CDFT-CI calculations[87], and the coupling element derivative. We then discuss the explicit and implicit contributions of constraint potential to the energy gradient in Sec II D.

\section{B. Assembling a matrix element/coupling derivative}

We will need to evaluate several expressions of similar form, so we step back from $W_{I J}^{x}$ and consider the general case of the matrix element of a one-electron (or zero-electron) operator $\hat{O}$ between two states $\left|\Phi_{I}\right\rangle$ and $\left|\Phi_{J}\right\rangle$, described in terms of the MO coefficients $\mathbf{c}_{I}$ and $\mathbf{c}_{J}$. Varying $\mathbf{c}_{I}$ freely can make the wavefunction $\left|\Phi_{I}\right\rangle$ non-normalized, which we correct for with an explicit normalization denominator.

$$
\begin{aligned}
M[\hat{O}] & =\frac{\left\langle\Phi_{I}|\hat{O}| \Phi_{J}\right\rangle}{\sqrt{\left\langle\Phi_{I} \mid \Phi_{I}\right\rangle\left\langle\Phi_{J} \mid \Phi_{J}\right\rangle}} \\
& =\frac{\operatorname{Tr}\left[\mathbf{c}_{I}^{\dagger} \mathbf{O} \mathbf{c}_{J}\left(\mathbf{c}_{I}^{\dagger} \mathbf{S} \mathbf{c}_{J}\right)^{-1}\right] \operatorname{det}\left(\mathbf{c}_{I}^{\dagger} \mathbf{S} \mathbf{c}_{J}\right)}{\sqrt{\operatorname{det}\left(\mathbf{c}_{I}^{\dagger} \mathbf{S c}_{I}\right) \operatorname{det}\left(\mathbf{c}_{J}^{\dagger} \mathbf{S c}_{J}\right)}}
\end{aligned}
$$

We will consider $\hat{O}=\left(\hat{V}_{I}+\hat{V}_{J}\right) / 2$, the case where there is no operator (so the matrix element is just an overlap), and $\hat{O}=\hat{w}$; we could also consider the gradient of the dipole moment of a CDFT-CI state with $\hat{O}=\hat{\mu}$, or the density gradient with $\hat{O}=\delta(\hat{\mathbf{x}})$, or other one-electron properties. We make $M$ variational with respect to the $\mathrm{MO}$ coefficients, writing

$$
M^{\mathrm{var}}=M[\hat{O}]-L\left(\mathbf{c}_{I}, \mathbf{t}_{I}\right)-L\left(\mathbf{c}_{J}, \mathbf{t}_{J}\right)
$$




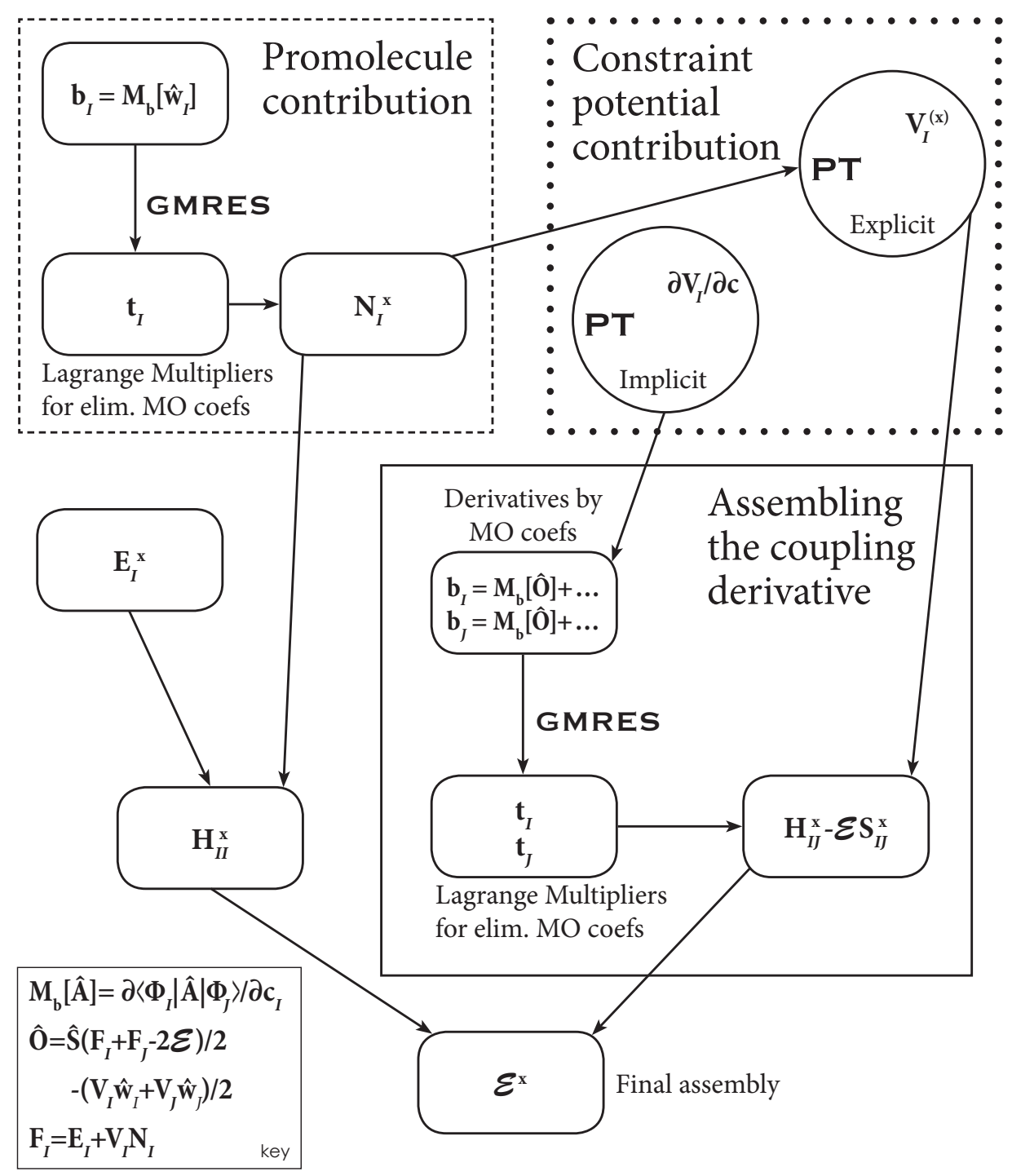

FIG. 1. The flowchart for evaluating the gradient of the CDFT-CI energy. The gradient of the promolecule-adjusted constraint values is computed as a variational matrix element (dashed box), using the GMRES linear solver to obtain the Lagrange multipliers needed for variationality. These $N_{I}^{x}$ are combined with the CDFT energy gradient to yield the diagonal elements of the Hamiltonian (bottom left), and also used to determine the explicit dependence of the constraint potential Lagrange multipliers on the nuclear coordinates, using a perturbation theory expression (upper right). A separate perturbation theory expression (also in the dotted box) gives the dependence of the constraint potential Lagrange multipliers on the MO coefficients (and thus the implicit dependence on nuclear position), which enters into the variationality of the coupling derivative (solid box), computed in a similar fashion to the promolecule contribution. With both diagonal and off-diagonal matrix elements available, the energy gradient is evaluated per Eq. (5) (bottom). 


\section{Lagrange multipliers to eliminate dependence of $M$ on the $M O$ coefficients}

The new term $L$ depends on Lagrange multipliers t to enforce variationality; there are $N_{\text {occ }} \times N_{\text {basis }}$ relevant MO coefficients, so there are $N_{\text {occ }} \times N_{\text {basis }}$ Lagrange multipliers $\mathbf{t}$, appearing as

$$
L(\mathbf{c}, \mathbf{t})=\operatorname{Tr}\left[\mathbf{t}^{\dagger} \cdot(\mathbf{F}[\tilde{\mathbf{P}}] \mathbf{c}-\mathbf{S c} \epsilon)\right]
$$

where $\tilde{\mathbf{P}}=3 \mathbf{P S P}-2 \mathbf{P S P S P}$ is the density matrix after McWeeny's purification transformation, [89] $\mathbf{P}=\mathbf{c c}^{\dagger}$ is the density matrix, and $\mathbf{S}$ remains the atomic orbital overlap matrix.

$\epsilon$ is a diagonal matrix of MO energies, which we define to be evaluated as $\epsilon_{i}=\frac{c_{i}^{\dagger} \mathbf{F} c_{i}}{c_{i}^{\dagger} \mathbf{S} c_{i}}$ so as to remain normalized when the orbitals themselves become unnormalized, per Eq. (12). We also introduce the Fock matrix $\mathbf{F}$, which has dependence on both the MO coefficients and the nuclear position, but we do not need to enforce that $\partial M^{\mathrm{var}} / \partial \mathbf{F}=0$. Eq. (14) is constructed such that the quantity in parentheses will always evaluate to zero when the system is at SCF convergence. Accordingly, $L$ will also always be zero at convergence, so $M^{\text {var }}$ will have the same value as $M$. Furthermore, $\partial M^{\text {var }} / \partial \mathbf{t}$ will also be zero by the self-consistency condition, which removes any need for gradients of $\mathbf{t}$ in evaluating chain-rule terms. (Note that the actual values of $\mathbf{t}$ are as-yet unspecified.) The McWeeny purified density matrix is required so that changes in the MO coefficients which do not preserve normalization do not affect the resulting Fock matrix; changes in normalization of the MO coefficients at first order will only affect the purified density matrix at the second order. No correction is needed for the MO coefficients that appear directly in $\mathbf{F} \mathbf{c}-\mathbf{S c} \epsilon$, as that expression is merely enforcing that the orbitals remain eigenvectors; a change in normalization does not affect that condition.

To enforce the variationality of $M^{\text {var }}$, we require

$$
\begin{aligned}
\frac{\partial M^{\mathrm{var}}}{\partial \mathbf{c}} & =0 \\
\Rightarrow \quad \frac{\partial M}{\partial \mathbf{c}} & =\frac{\partial L(\mathbf{c}, \mathbf{t})}{\partial \mathbf{c}},
\end{aligned}
$$

for derivatives with respect to both $\mathbf{c}_{I}$ and $\mathbf{c}_{J}$. We expand the two sides of this equation 
separately:

$$
\begin{aligned}
\frac{\partial M}{\partial \mathbf{c}_{I}}= & \operatorname{det}\left(\mathbf{c}_{I}^{\dagger} \mathbf{S} \mathbf{c}_{J}\right) \mathbf{O} \mathbf{c}_{J}\left(\mathbf{c}_{I}^{\dagger} \mathbf{S} \mathbf{c}_{J}\right)^{-1}-\mathbf{S} \mathbf{c}_{J}\left(\mathbf{c}_{I}^{\dagger} \mathbf{S} \mathbf{c}_{J}\right)^{-1} \mathbf{c}_{I}^{\dagger} \mathbf{O} \mathbf{c}_{J}\left(\mathbf{c}_{I}^{\dagger} \mathbf{S} \mathbf{c}_{J}\right)^{-1} \\
& +M[\hat{O}] \mathbf{S} \mathbf{c}_{J}\left(\mathbf{c}_{I}^{\dagger} \mathbf{S c}_{J}\right)^{-1}-M[\hat{O}] \mathbf{S} \mathbf{c}_{I} \\
\frac{\partial L(\mathbf{c}, \mathbf{t})}{\partial c_{\mu i}}= & t_{\nu j} \frac{\partial F_{\nu \lambda}[\tilde{\mathbf{P}}]}{\partial c_{\mu i}} c_{\lambda j}+t_{\nu i} F_{\nu \mu}[\tilde{\mathbf{P}}]-t_{\nu i} S_{\nu \mu} \epsilon_{i}-t_{\nu j} S_{\nu \delta} c_{\lambda j} \frac{\partial F_{\lambda \sigma}[\tilde{\mathbf{P}}]}{\partial c_{\mu i}} c_{\sigma j} c_{\delta j} \\
& -2 t_{\nu i} S_{\nu \delta} F_{\mu \sigma}[\tilde{\mathbf{P}}] c_{\sigma i} c_{\delta i}+2 t_{\nu i} S_{\nu \delta} c_{\lambda i} F_{\lambda \sigma}[\tilde{\mathbf{P}}] c_{\sigma i} S_{\mu \gamma} c_{\gamma i} c_{\delta i} .
\end{aligned}
$$

The overall structure of the expression reduces to

$$
M_{b}[\hat{O}] \equiv \frac{\partial M}{\partial c_{\mu i}}=b_{\mu i}=A_{\mu i}^{\nu j} t_{\nu j}
$$

where we have adopted explicit indices and the Einstein summation convention and defined a new quantity $M_{b}[\hat{O}]$ for future use.[90] The final line, however, makes it clear that this is a linear system which can be solved for $\mathbf{t}$. The $\mathbf{A}$ matrix that defines this linear system is of size $\left(N_{\text {occ }} \cdot N_{\text {virt }}\right) \times\left(N_{\text {occ }} \cdot N_{\text {virt }}\right)$ which requires $O\left(N^{4}\right)$ storage and $O\left(N^{6}\right)$ time for a direct inversion, a step backwards from Eq. (10). However, we can solve the linear system in Eq. (19) without constructing $\mathbf{A}$, by using an iterative linear solver. This allows us to leverage

the fact that the product $A_{\mu i}^{\nu j} t_{\nu j}$ may be evaluated efficiently without computing $\mathbf{A}$. The explicit expression for the contraction is given by

$$
\begin{aligned}
A_{\mu i}^{\nu j} t_{\nu j} & =A_{0}{ }_{\mu i} t_{\nu j}+\frac{\partial F_{\nu \lambda}[\tilde{\mathbf{P}}]}{\partial c_{\mu i}} Q_{\lambda \nu}[\mathbf{t}] \\
A_{0 \mu i}{ }_{\mu i} t_{\nu j} & =t_{\nu j} \delta_{i j}\left(F_{\nu \mu}[\tilde{\mathbf{P}}]-S_{\nu \mu} \epsilon_{j}-2 S_{\nu \delta} F_{\mu \sigma}[\tilde{\mathbf{P}}] c_{\sigma j} c_{\delta j}+S_{\nu \delta} c_{\lambda j} F_{\lambda \sigma}[\tilde{\mathbf{P}}] c_{\sigma j} S_{\mu \gamma} c_{\gamma j} c_{\delta j}\right),
\end{aligned}
$$

where we define the pseudo-density matrix

$$
Q_{\lambda \nu}=c_{\lambda i} t_{\nu i}-c_{\lambda i} t_{\alpha i} S_{\alpha \beta} c_{\beta i} c_{\nu i}
$$

We have also separated $\mathbf{A}$ into a term $\mathbf{A}_{0}$ and terms dependent on $\partial \mathbf{F} / \partial \mathbf{c}$. If considered as a matrix, $\mathbf{A}_{0}$ is block-diagonal - each orbital only interacts with the corresponding "orbital" from $t$. In other words, all of the terms in $A_{0}$ include a Kronecker delta $\delta_{i j}$, so there is only $O\left(N^{3}\right)$ work to be done in the overall multiplication.

We have left unexpanded the expression $\partial F_{\lambda \sigma}[\tilde{\mathbf{P}}] / \partial c_{\mu i}$, a quantity whose determination is complicated by the use of the purified density $\tilde{\mathbf{P}}$. Performing this computation requires a 
breakdown of the different contributions to $\mathbf{F}$, with the Coulomb integrals and Hartree-Fock exchange being treated differently from the DFT exchange and correlation (XC) functionals. (The one-electron Hamiltonian of course has no dependence on the MO coefficients.) Bearing in mind our need to compute only the product $\mathbf{A} t$ and not $\mathbf{A}$ itself, we examine the contraction of some pseudo-density-matrix $Q_{\lambda \sigma}$ against $\partial F_{\lambda \sigma}[\tilde{\mathbf{P}}] / \partial c_{\delta i}$, looking at each of these terms in turn.

$$
\begin{aligned}
\frac{\partial J_{\lambda \sigma}[\tilde{\mathbf{P}}]}{\partial c_{\delta i}} Q_{\lambda \sigma} & =2 J_{\mu \delta}[\mathbf{Q}] P_{\mu \alpha} S_{\alpha \beta} c_{\beta i}+2 S_{\delta \beta} P_{\beta \nu} J_{\mu \nu}[\mathbf{Q}] c_{\mu i} \\
& -4 S_{\delta \eta} P_{\eta \nu} J_{\mu \nu}[\mathbf{Q}] P_{\mu \alpha} S_{\alpha \beta} c_{\beta i}
\end{aligned}
$$

where we use the fact that $\mathbf{P S P}=\mathbf{P}$ at convergence. This requires only a single Coulomb build from the pseudo-density $\mathbf{Q}$, and matrix multiplications with $\mathbf{S}$ and $\mathbf{P}$.

In a similar fashion, the expression for the exchange derivative becomes

$$
\begin{aligned}
\frac{\partial K_{\lambda \sigma}[\tilde{\mathbf{P}}]}{\partial c_{\delta i}} Q_{\lambda \sigma} & =2 K_{\mu \delta}[\mathbf{Q}] P_{\mu \alpha} S_{\alpha \beta} c_{\beta i}+2 S_{\delta \beta} P_{\beta \nu} K_{\mu \nu}[\mathbf{Q}] c_{\mu i} \\
& -4 S_{\delta \eta} P_{\eta \nu} K_{\mu \nu}[\mathbf{Q}] P_{\mu \alpha} S_{\alpha \beta} c_{\beta i}
\end{aligned}
$$

The DFT contributions are not quite as straightforward, since the XC matrix (for a pure functional) is more properly written as

$$
v_{x c}=v_{x c}[\rho(\tilde{\mathbf{P}})]
$$

From the chain rule,

$$
\begin{aligned}
\frac{\partial v_{x c}}{\partial c_{\delta i}} & =\frac{\partial v_{x c}}{\partial \rho} \frac{\partial \rho}{\partial \tilde{\mathbf{P}}} \frac{\partial \tilde{\mathbf{P}}}{\partial c_{\delta i}} \\
& =\left(\frac{\partial v_{x c}}{\partial \rho} \frac{\partial \rho}{\partial \tilde{\mathbf{P}}}\right) \frac{\partial \tilde{\mathbf{P}}}{\partial c_{\delta i}}
\end{aligned}
$$

The quantity in parentheses is the implicit first derivative of the XC matrix, which is generally only used by being contracted against a "trial density", as we are doing here as we contract against $\mathbf{Q}$. Thus,

$$
\begin{aligned}
\frac{\partial\left(v_{x c}\right)_{\lambda \sigma}}{\partial c_{\delta i}} Q_{\lambda \sigma} & =\left(Q_{\lambda \sigma} \frac{\partial\left(v_{x c}\right)_{\lambda \sigma}}{\partial \rho} \frac{\partial \rho}{\partial \tilde{P}_{\alpha \beta}}\right) \frac{\partial \tilde{P}_{\alpha \beta}}{\partial c_{\delta i}} \\
& =X_{\alpha \beta} \frac{\partial \tilde{P}_{\alpha \beta}}{\partial c_{\delta i}} \\
& =2 X_{\delta \mu} P_{\mu \nu} S_{\nu \lambda} c_{\lambda i}+2 S_{\delta \mu} P_{\mu \nu} X_{\nu \lambda} c_{\lambda i}-4 S_{\delta \mu} P_{\mu \nu} X_{\nu \lambda} P_{\lambda \sigma} S_{\sigma \eta} c_{\eta i}
\end{aligned}
$$


in an analogous fashion to the coulomb and exchange terms. We implicitly define the quantity $X_{\alpha \beta}$ as the contraction of $\mathbf{Q}$ against the implicit first derivative of the $\mathrm{XC}$ matrix. The structure of equation (29) parallels equations (23) and (24), with the implicit first derivative of the $\mathrm{XC}$ matrix taking the place of the two-electron integrals. All of these terms (Coulomb, exchange, and DFT) may be efficiently evaluated in $O\left(N^{3}\right)$ time or less and $O\left(N^{2}\right)$ space.

\section{Iterative linear solver (GMRES)}

Now that the matrix-vector product is available, we can proceed to the iterative linear solver. We have implemented the GMRES (Generalized Minimum Residual) algorithm in Q-CHEM; the algorithm is covered extensively elsewhere, [91, 92] but we give a brief summary here. The goal is to construct an approximate solution to the linear system

$$
\mathbf{A} \cdot \mathbf{x}=\mathbf{b}
$$

without explicitly operating on the (square) matrix A, instead only evaluating matrix-vector products $\mathbf{A} \cdot \mathbf{t}_{i}$. The expectation is that an approximate solution with sufficiently small residual can be obtained in a constant number of iterations, essentially independent of the dimension of $\mathbf{A}$. For the systems we consider here, that constant is around twenty GMRES iterations. We use the (left) preconditioned form of GMRES:

$$
\left(\mathbf{A}_{0}^{-1} \mathbf{A}\right) \cdot \mathbf{t}=\mathbf{A}_{0}^{-1} \cdot \mathbf{b}
$$

where $\mathbf{A}_{0}$ is an easily inverted approximation to $\mathbf{A}$. There is a block-diagonal component to our $\mathbf{A}$ (the $\mathbf{A}_{0}$ of equation (20)), which in general is much larger in magnitude than the contribution coming from contractions against the derivative of the Fock matrix. This blockdiagonal term could be explicitly constructed with $O\left(N^{3}\right)$ effort and blockwise inverted for $O\left(N^{4}\right)$ effort, but we can retain $O\left(N^{3}\right)$ time by only considering the first two terms of $\mathbf{A}_{0}$,

$$
A_{0 \mu i}^{\prime \nu j}=\delta_{i j}\left(F_{\mu \nu}-S_{\mu \nu} \epsilon_{j}\right)
$$

The inversion of $\mathbf{A}_{0}^{\prime}$ is equivalent to solving the systems

$$
\left(\mathbf{F}-\mathbf{S} \epsilon_{i}\right) \mathbf{t}_{i}=\mathbf{b}_{i}
$$


which does not necessarily involve explicitly constructing $\mathbf{A}_{0}^{\prime-1}$ in matrix form. Conceptually, this is effected by transforming to the MO basis, where $\mathbf{F}$ and $\mathbf{S}$ are diagonal, so the inversion is trivial. However, since $\left(\mathbf{F}-\mathbf{S} \epsilon_{i}\right)^{-1}$ is singular at $\epsilon_{i}$, we use the pseudo-inverse which is justified below. After some algebra, the solution is

$$
t_{\mu i}=\frac{1}{\epsilon_{p}-\epsilon_{i}} c_{\mu p} c_{\nu p} b_{\nu i} \quad(p \neq i) .
$$

The actual determination of (the approximation solution for) $\mathbf{x}$ involves just matrix-matrix products, yielding the desired $O\left(N^{3}\right)$ time. Since the energy denominator is only nonzero when $p \neq i$, this preconditioner will not treat components of $b_{\mu i}$ which are proportional to actual orbitals $c_{\mu i}$; however, these components are zero by construction, having been eliminated by the normalization denominator of Eq. (12).

\section{Assembling the coupling derivative}

Having determined (via GMRES) the Lagrange multipliers $\mathbf{t}$ which make the function $M^{\mathrm{var}}$ of Eq. (13) variational with respect to the MO coefficients, we now return to the chain rule and use them. The terms from $\partial M^{\mathrm{var}} / \partial \mathbf{t}$ have already been shown to be zero, but our definition of $L$ also introduced a dependence on $\mathbf{F}$ to the full $M^{\text {var }}$ which must be included when applying the chain rule, so that

$$
\frac{d M^{\mathrm{var}}}{d x}=\frac{\partial M^{\mathrm{var}}}{\partial \mathbf{O}} \frac{d \mathbf{O}}{d x}+\frac{\partial M^{\mathrm{var}}}{\partial \mathbf{S}} \frac{d \mathbf{S}}{d x}+\frac{\partial M^{\mathrm{var}}}{\partial \mathbf{F}} \frac{d \mathbf{F}}{d x}
$$

To evaluate these chain-rule terms, we repartition this expression as

$$
\frac{d M}{d x}=\frac{d M^{\mathrm{var}}}{d x}=\tilde{M}_{x}[\hat{O}]-\tilde{L}_{x}\left(\mathbf{c}_{I}, \mathbf{t}_{I}\right)-\tilde{L}_{x}\left(\mathbf{c}_{J}, \mathbf{t}_{J}\right),
$$

where the tilde indicates to exclude the terms involving $\partial \mathbf{c} / \partial x$.

$$
\begin{aligned}
\tilde{M}_{x}[\hat{O}]=\operatorname{Tr} & {\left[\left\{\mathbf{c}_{I}^{\dagger} \mathbf{O}^{x} \mathbf{c}_{J}+\mathbf{c}_{I}^{\dagger} \mathbf{O} \mathbf{c}_{J}\left(\mathbf{c}_{I}^{\dagger} \mathbf{S} \mathbf{c}_{J}\right)^{-1}\left(\mathbf{c}_{I}^{\dagger} \mathbf{S}^{x} \mathbf{c}_{J}\right)\right\}\left(\mathbf{c}_{I}^{\dagger} \mathbf{S} \mathbf{c}_{J}\right)^{-1}\right] \operatorname{det}\left(\mathbf{c}_{I}^{\dagger} \mathbf{S} \mathbf{c}_{J}\right) } \\
& +M[\hat{O}] \cdot \operatorname{Tr}\left[\left(\mathbf{c}_{I}^{\dagger} \mathbf{S} \mathbf{c}_{J}\right)^{-1}\left(\mathbf{c}_{I}^{\dagger} \mathbf{S}^{x} \mathbf{c}_{J}\right)\right] \\
& -\frac{1}{2} M[\hat{O}] \operatorname{Tr}\left[\mathbf{c}_{I}^{\dagger} \mathbf{S}^{x} \mathbf{c}_{I}+\mathbf{c}_{J}^{\dagger} \mathbf{S}^{x} \mathbf{c}_{J}\right] .
\end{aligned}
$$

and

$$
\tilde{L}_{x}(\mathbf{c}, \mathbf{t})=\operatorname{Tr}\left[\mathbf{t}^{\dagger} \cdot\left(\mathbf{F}^{(x)}[\tilde{\mathbf{P}}] \mathbf{c}-\mathbf{S}^{x} \mathbf{c} \epsilon-\mathbf{S c} \epsilon^{(x)}\right)\right]
$$


where

$$
\begin{aligned}
\epsilon_{p}^{(x)} & =\frac{c_{\mu p} F_{\mu \nu}^{(x)}[\tilde{\mathbf{P}}] c_{\nu p}}{c_{\alpha p} S_{\alpha \beta} c_{\beta p}}-\frac{c_{\mu p} F_{\mu \nu}[\tilde{\mathbf{P}}] c_{\nu p}}{\left(c_{\alpha p} S_{\alpha \beta} c_{\beta p}\right)^{2}} c_{\lambda p} S_{\lambda \sigma}^{x} c_{\sigma p} \\
& =c_{\mu p} F_{\mu \nu}^{(x)}[\tilde{\mathbf{P}}] c_{\nu p}-\epsilon_{p} c_{\mu p} S_{\mu \nu}^{x} c_{\nu p} \\
& =c_{\mu p}\left(F_{\mu \nu}^{(x)}[\tilde{\mathbf{P}}]-S_{\mu \nu}^{x} \epsilon_{p}\right) c_{\nu p}
\end{aligned}
$$

and $\mathbf{F}^{(x)}[\tilde{\mathbf{P}}]$ is the partial derivative of the Fock matrix including the McWeeny purification of the density matrix (but excluding the position dependence of the MO coefficients). That is,

$$
\begin{aligned}
\mathbf{F}^{(x)}[\tilde{\mathbf{P}}] & =\mathbf{F}^{(x)}[\mathbf{P}]+\mathbf{J}\left[\tilde{\mathbf{P}}^{(x)}\right]+c_{K} \mathbf{K}\left[\tilde{\mathbf{P}}^{(x)}\right]+\left(\frac{\partial\left(v_{x c}\right)}{\partial \rho} \frac{\partial \rho}{\partial \tilde{\mathbf{P}}}\right) \tilde{\mathbf{P}}^{(x)} \\
\tilde{\mathbf{P}}^{(x)} & =3 \mathbf{P} \mathbf{S}^{x} \mathbf{P}-2 \mathbf{P} \mathbf{S}^{x} \mathbf{P S P}-2 \mathbf{P S P S}^{x} \mathbf{P} \\
& =-\mathbf{P S}^{x} \mathbf{P} .
\end{aligned}
$$

The DFT contribution is again an instance of the implicit first derivative of the XC matrix used in Eq. (29), $\mathbf{F}^{(x)}[\mathbf{P}]$ is just the standard partial derivative of the Fock matrix with respect to the nuclear position, and $c_{K}$ represents the coefficient of exact exchange in the density functional.

To avoid explicitly computing and storing $\mathbf{F}^{(x)}[\tilde{\mathbf{P}}]$ (which is expensive), we contract pseudo density matrices $\mathbf{X}$ (defined below) for the exchange and Coulomb builds instead of the many $\tilde{\mathbf{P}}^{(x)}$. Equation (37) then becomes

$$
\begin{aligned}
\tilde{L}_{x}(\mathbf{c}, \mathbf{t}) & =t_{\mu p} F_{\mu \nu}^{(x)}[\tilde{\mathbf{P}}] c_{\nu p}-t_{\mu p} S_{\mu \nu}^{x} c_{\nu p} \epsilon_{p}-t_{\mu p} S_{\mu \nu} c_{\nu p} c_{\lambda p} F_{\lambda \sigma}^{(x)}[\tilde{\mathbf{P}}] c_{\sigma p}+t_{\mu p} S_{\mu \nu} c_{\nu p} c_{\lambda p} S_{\lambda \sigma}^{x} c_{\sigma p} \epsilon_{p} \\
& =t_{\mu p} F_{\mu \nu}^{(x)}[\tilde{\mathbf{P}}] c_{\nu p}-t_{\lambda p} S_{\lambda \sigma} c_{\sigma p} c_{\mu p} F_{\mu \nu}^{(x)}[\tilde{\mathbf{P}}] c_{\nu p}-t_{\mu p} S_{\mu \nu}^{x} c_{\nu p} \epsilon_{p}+t_{\lambda p} S_{\lambda \sigma} c_{\sigma p} c_{\mu p} S_{\mu \nu}^{x} c_{\nu p} \epsilon_{p} \\
& =F_{\mu \nu}^{(x)}[\tilde{\mathbf{P}}]\left(t_{\mu p} c_{\nu p}-t_{\lambda p} S_{\lambda \sigma} c_{\sigma p} c_{\mu p} c_{\nu p}\right)-S_{\mu \nu}^{x}\left(t_{\mu p} c_{\nu p}+t_{\lambda p} S_{\lambda \sigma} c_{\sigma p} c_{\mu p} c_{\nu p}\right) \epsilon_{p} \\
& =F_{\mu \nu}^{(x)}[\tilde{\mathbf{P}}] X_{\nu \mu}-S_{\mu \nu}^{x} Y_{\nu \mu} \\
& =\operatorname{Tr}\left[\tilde{\mathbf{F}}{ }^{(x)}[\mathbf{P}] \mathbf{X}+\left\{\mathbf{J}[\mathbf{X}]+c_{k} \mathbf{K}[\mathbf{X}]+\left(\frac{\partial\left(v_{x c}\right)}{\partial \rho} \frac{\partial \rho}{\partial \tilde{\mathbf{P}}} \mathbf{X}\right)\right\} \tilde{\mathbf{P}}^{(x)}-\mathbf{S}^{x} \mathbf{Y}\right]
\end{aligned}
$$

where we have defined

$$
\begin{aligned}
X_{\nu \mu} & =t_{\mu p} c_{\nu p}-t_{\lambda p} S_{\lambda \sigma} c_{\sigma p} c_{\mu p} c_{\nu p} \\
Y_{\nu \mu} & =\left(t_{\mu p} c_{\nu p}-t_{\lambda p} S_{\lambda \sigma} c_{\sigma p} c_{\mu p} c_{\nu p}\right) \epsilon_{p}
\end{aligned}
$$

Together, these terms efficiently evaluate the gradient of a single matrix element, but equations (17)-(19) imply that the actual values of $\mathbf{t}_{I}$ depend on the $J$ state for the matrix 
element in question - there is no efficiency gained by evaluating multiple matrix elements at the same time, and we must compute $\mathbf{X}_{I}^{(J)}$ and the corresponding Fock-like matrix separately for each pair of states (note that $\mathbf{X}_{I}^{(J)} \neq \mathbf{X}_{J}^{(I)}$ ). This remains more efficient than working with the Fock matrix gradients directly because the number of states in the CI matrix will be small, even when the number of atoms in the calcuation is large; it also allows us to keep the storage required at the $O\left(N^{2}\right)$ level.

It should also be noted that, as written, $\mathbf{X}_{I}^{(J)}$ is in general asymmetric, resulting in asymmetric $\mathbf{J}[\mathbf{X}]$, etc. Since it is easy to show that $\operatorname{Tr}\left[\mathbf{J}[\mathbf{X}] \tilde{\mathbf{P}}^{(x)}\right]=\operatorname{Tr}\left[\mathbf{J}\left[\left(\mathbf{X}+\mathbf{X}^{\dagger}\right) / 2\right] \tilde{\mathbf{P}}^{(x)}\right]$ for symmetric $\tilde{\mathbf{P}}^{(x)}$, we symmetrize $\mathbf{X}_{I}^{(J)}$ for convenience of calculation.

The procedure to obtain $d M / d x=d M^{\mathrm{var}} / d x$ then is to determine $\mathbf{b}_{I}$ and $\mathbf{b}_{J}$ using the $M_{b}[\hat{O}]$ formula, and perform two GMRES calculations (using the appropriate A matrix for state $I$ or $J$ ) to determine the Lagrange multipliers $\mathbf{t}_{I}$ and $\mathbf{t}_{J}$. Then, $\tilde{M}_{x}[\hat{O}]$ from Eq. (36) and $\tilde{L}_{x}(\mathbf{c}, \mathbf{t})$ from Eq. (42) are substituted into Eq. (35) to obtain the final gradient.

It bears reiterating that $\tilde{L}_{x} \neq d L / d x$, since it omits the $\partial L / \partial \mathbf{c}$ terms which only cancel when the full quantity $d M / d x$ is being evaluated. This is why $\mathbf{t}$ must be redetermined for each operator and for each state.

\section{Promolecule contribution}

Having established the general form for evaluating the gradient of a matrix element of a one-electron operator between two distinct states, we now step back and note a particular issue with the formulation of CDFT-CI which makes the computation of $\mathcal{E}^{x}$ (Eq. (5)) more complicated. Recall that the CDFT equations involve minimizing the value of $E[\rho]+\sum_{k} V_{k}\left(\int \hat{w}_{k} \rho d^{3} r-N_{k}\right)$ with respect to $\rho$ and maximizing with respect to $V_{k}$ for fixed $\hat{w}_{k}$ and $N_{k}$ (Eq. 1). However, when adapting CDFT for use in CDFT-CI, the concept of a "promolecule density" was introduced which produced modified values of $N_{k}$ for a given system.[87] This used the converged density from independent calculations on noninteracting fragments, in conjunction with $\hat{w}_{k}$, to produce new values of $N_{k}$. Therefore, $N_{k}$ also depends on the nuclear position since it is clear that the converged density of non-interacting fragments has a dependence on nuclear position (when there is more than one atom in a fragment). This dependence will in turn trickle through to affect the other properties of the 
system, such as the free energy of state $I$

$$
F_{I}=E_{I}+\sum_{k} V_{I, k} N_{I, k}
$$

in the presence of constraints. Considering the above fact, the free energy gradient becomes

$$
F_{I}^{x}=E_{I}^{x}+\sum_{k} V_{I, k} N_{I, k}^{x}
$$

Thus, in equation (7) when we said that $H_{I I}^{x}$ is the gradient of the CDFT state free energy, it is the gradient of that energy provided that the constraint values are also changing according to the promolecule formalism, i.e., it is the gradient including this correction of Eq. (46).

Recalling the definition of $N_{k}$ :

$$
N_{k}=\left\langle\Phi_{I}\left|\hat{w}_{k}\right| \Phi_{I}\right\rangle
$$

we note that the expression is precisely the matrix element of a one-electron operator, so we can reuse wholesale the algebraic machinery developed for $M[\hat{O}]$ in Sec. II B 3 to obtain $N_{k}^{x}$, with $\mathbf{b}_{k}=2 M_{b}\left[\hat{w}_{k}\right]$ yielding Lagrange multipliers $\mathbf{t}_{k}$ via GMRES, and $N_{k}^{x}=\tilde{M}_{x}\left[\hat{w}_{k}\right]-$ $\tilde{L}_{x}\left(\mathbf{c}, \mathbf{t}_{k}\right)$. That $N_{k}$ is a matrix element between two identical states serves only to simplify the algebra in that only one set of Lagrange multipliers is needed and $\partial N_{k} / \partial \mathbf{c}=2 M_{b}\left[\hat{w}_{k}\right]$ due to the symmetry. It should be noted that when evaulating $\partial M\left[\hat{w}_{k}\right] / \partial \mathbf{c}$, the isolation between independent fragments must be retained.

The gradient of the weight operator (for the full system, not the isolated fragments) is needed to evaluate $\tilde{M}_{x}\left[\hat{w}_{k}\right]$; for the Becke weights used in this implementation of CDFT-CI, such gradient terms have been computed in Reference [93].

\section{Constraint potential contribution}

While the single-state values of $N_{k}^{x}$ were sufficient to correct $W_{I I}^{x}$ as in Eq. (46), the

off-diagonal elements $W_{I J}^{x}$ include the matrix element of the constraint potential $\hat{V}_{k}$ between two different states, and those quantities cannot be derived solely from single-state values of $N_{k}^{x}$.

Given that the overall gradient of the constraint potential is

$$
\hat{V}_{k}^{x}=V_{k} \hat{w}_{k}^{x}+V_{k}^{x} \hat{w}_{k}
$$


and the gradients of the weight matrices, $\mathbf{w}_{k}^{x}$, were used in evaluating $\tilde{M}_{x}\left[\hat{w}_{k}\right]$, it remains only to determine the gradient of the constraint potential Lagrange multipliers, $V_{k}^{x}$. These are intrinsically linked to the constraint value gradients $N_{k}^{x}$, changing in lockstep to maintain the solution to the inner SCF procedure. As such, we require a mechanism to go from the promolecule-derived constraint value gradients $N_{k}^{x}$ to the gradients of the constraint potential Lagrange multipliers, $V_{k}^{x}$. It becomes clear that over the course of the entire double SCF

calculation, the Lagrange multipliers $V_{k}$ depend on nuclear coordinates both "directly", through the explicit dependence of the Fock matrix (without constraint potentials) and AO overlap on the nuclear coordinates, and also indirectly, through the dependence of the Fock matrix on the MO coefficients (which in turn depend on the nuclear coordinates). It proves convenient to separate these dependencies, pushing the implicit contribution back into the b vector of Eq. (19) for $\mathbf{W}$, and only treating the explicit dependence at this junction.

\section{Explicit contribution}

Treating just the explicit contribution requires holding the MO coefficients used to build the Fock matrix fixed (while varying the nuclear geometry to $x+\delta x$ ), essentially just limiting the calculation to a single cycle of the outer SCF loop. The resulting change in the constraint potential Lagrange multipliers $\delta V_{k}$ are the quantities we need for the explicit contribution. Because we consider only explicit changes in the Fock matrix (ignoring its nonlinear dependence on the MO coefficients), the resulting changes to the orbitals can be determined solely via perturbation theory. The derivation is given in Appendix A, with the main result being:

$$
\begin{aligned}
N_{k}^{x}-\left\langle\Phi\left|\hat{w}_{k}^{x}\right| \Phi\right\rangle+\sum_{i}\left\langle\phi_{i}\left|w_{k}\right| \phi_{i}\right\rangle\left\langle\phi_{i}\left|\hat{S}^{x}\right| \phi_{i}\right\rangle & \\
-2 \sum_{i \neq p}\left\langle\phi_{i}\left|\hat{w}_{k}\right| \phi_{p}\right\rangle \frac{\left\langle\phi_{p}\left|\hat{F}^{(x)}-\epsilon_{i} \hat{S}^{x}+\sum_{l} V_{l} \hat{w}_{l}^{x}\right| \phi_{i}\right\rangle}{\epsilon_{i}-\epsilon_{p}} & =2 \sum_{l} \sum_{i \neq p}\left\langle\phi_{i}\left|\hat{w}_{k}\right| \phi_{p}\right\rangle \frac{\left\langle\phi_{p}\left|\hat{w}_{l}\right| \phi_{i}\right\rangle}{\epsilon_{i}-\epsilon_{p}} V_{l}^{x} \\
b_{k} & =A_{k}^{l} V_{l}^{x},
\end{aligned}
$$

Since the promolecule specification requires both charge and spin constraints on a given fragment, there will in general be at least two constraints, and thus a linear system to be solved. Fortunately, the A matrix does not have any position dependence and can be precomputed and inverted just once, with $b$ (and thus $V_{l}^{x}$ ) being computed for a single 
nuclear coordinate at a time. The $b$ vector contains the gradient of the weight matrix and the gradient of the overlap, which have already been computed, and also $\mathbf{F}^{(x)}[\tilde{\mathbf{P}}]$, which we have thus far avoided using explicitly (in Eq.(42)). The reasons for doing so remain valid, so we again need to reformulate the fourth term in the left hand side of Eq.(49) in terms of some pseudo-density matrix. Noting that this expression is written in the MO basis, the Fock contribution of this term is given by

$$
\begin{aligned}
-2 \sum_{i \neq p} w_{i p}^{k} \frac{F_{p i}^{(x)}[\tilde{\mathbf{P}}]}{\epsilon_{i}-\epsilon_{p}} & =-2 \sum_{i \neq p} w_{i p}^{k} \frac{c_{\mu p} c_{\nu i} F_{\mu \nu}^{(x)}[\tilde{\mathbf{P}}]}{\epsilon_{i}-\epsilon_{p}} \\
& =-2 F_{\mu \nu}^{(x)}[\tilde{\mathbf{P}}] \tilde{w}_{\nu \mu}^{k}
\end{aligned}
$$

in the AO basis, where we have defined

$$
\tilde{w}_{\nu \mu}^{k}=\sum_{p} \sum_{i \neq p} c_{\nu i} \frac{w_{i p}^{k}}{\epsilon_{i}-\epsilon_{p}} c_{\mu p} .
$$

Hence, we can use exactly the same machinery as we did in Eq.(42).

\section{Implicit dependence}

Having constructed an expression for the explicit dependence of the constraint potential on the nuclear position, in the form of an expression $(\delta V) \hat{w}+V(\delta \hat{w})$ which is added to the gradient of the Fock matrix, it remains to treat the implicit dependence, which enters through the MO coefficients used to build the Fock matrix. As previously indicated, this will be included through the Lagrange multipliers in $L(\mathbf{c}, \mathbf{t})$, by adding an extra contribution from $\partial V / \partial \mathbf{c}$ to the $\mathbf{b}$ vector in Eq. (19). This $\partial V / \partial \mathbf{c}$ contribution is obtained from a perturbation theory calculation using a very similar structure to that of the $\partial V / \partial x$ contribution above, including the need for a linear system in the various constraints. In this case the perturbation is now $\delta \hat{F}=\partial \hat{F} / \partial \mathbf{c}$ and $\delta V_{l}=\partial V_{l} / \partial \mathbf{c}$. The form of the equations is identical to Eq. (49) with simplifications that $\delta N_{k}$ is zero (the target constraint value does not depend on the MO

coefficients passed to the Fock matrix), and $\delta \hat{S}$ and $\delta \hat{w}$ are also zero. The orbital overlap then becomes just

$$
\left\langle\phi_{p} \mid \delta \phi_{i}\right\rangle=\frac{\left\langle\phi_{p}\left|\delta F+\sum_{l}\left(\delta V_{l}\right) \hat{w}_{l}\right| \phi_{i}\right\rangle}{\epsilon_{i}-\epsilon_{p}}
$$


And the linear system to be solved:

$$
\begin{aligned}
-\sum_{i \neq p}\left\langle\phi_{i}\left|\hat{w}_{k}\right| \phi_{p}\right\rangle \frac{\left\langle\phi_{p}|\delta \hat{F}| \phi_{i}\right\rangle}{\epsilon_{i}-\epsilon_{p}} & =\sum_{l} \sum_{i \neq p}\left\langle\phi_{i}\left|\hat{w}_{k}\right| \phi_{p}\right\rangle \frac{\left\langle\phi_{p}\left|\hat{w}_{l}\right| \phi_{i}\right\rangle}{\epsilon_{i}-\epsilon_{p}}\left(\delta V_{l}\right), \\
b_{k} & =A_{k}^{l}\left(\delta V_{l}\right)
\end{aligned}
$$

The A matrix is identical to the one in Eq. (49). All of the $b$ vectors may be generated at once as contractions against $\partial F / \partial \mathbf{c}$, repeated for the number of constraints applied to the system. Such contractions against $\partial F / \partial \mathbf{c}$ were described in Eqs. (23) through (29).

\section{E. Final assembly}

At this point, all the pieces are in place to compute $(\mathbf{H}-\mathcal{E} \mathbf{S})_{I J}^{x}=W_{I J}^{x}$, the last remaining

piece before Eq. (5) may be applied to obtain $\mathcal{E}^{x}$. In the now-familiar procedure, we construct

$$
W_{I J}^{\mathrm{var}}=\frac{W_{I J}}{\sqrt{\left\langle\Phi_{I} \mid \Phi_{I}\right\rangle\left\langle\Phi_{J} \mid \Phi_{J}\right\rangle}}-L\left(\mathbf{c}_{I}, \mathbf{t}_{I}\right)-L\left(\mathbf{c}_{J}, \mathbf{t}_{J}\right)
$$

and solve for the Lagrange multipliers $\mathbf{t}_{I}$ and $\mathbf{t}_{J}$ which make $W_{I J}^{\mathrm{var}}$ variational with respect to the MO coefficients. To do so, we need vectors $\mathbf{b}_{I}$ and $\mathbf{b}_{J}$ as input for GMRES calculations to determine $\mathbf{t}_{I}$ and $\mathbf{t}_{J}$; in our formalism the contribution from $\mathbf{H}$ is split out into terms arising from the constrained states, so this really looks like

$$
\begin{aligned}
& \frac{\partial(\mathbf{H}-\mathcal{E} \mathbf{S})_{I J}}{\partial \mathbf{c}_{I}} \\
= & \frac{\partial}{\partial \mathbf{c}_{I}}\left[\frac{\left(\frac{F_{I}+F_{J}}{2} S_{I J}-\left\langle\Phi_{I}\left|\frac{\hat{V}_{I}+\hat{V}_{J}}{2}\right| \Phi_{J}\right\rangle-\mathcal{E} S_{I J}\right)}{\sqrt{\left\langle\Phi_{I} \mid \Phi_{I}\right\rangle\left\langle\Phi_{J} \mid \Phi_{J}\right\rangle}}\right] \\
= & -\frac{\partial}{\partial \mathbf{c}_{I}}\left[\frac{\left\langle\Phi_{I}\left|\frac{\hat{V}_{I}+\hat{V}_{J}}{2}\right| \Phi_{J}\right\rangle}{\sqrt{\left\langle\Phi_{I} \mid \Phi_{I}\right\rangle\left\langle\Phi_{J} \mid \Phi_{J}\right\rangle}}\right]+\left(\frac{F_{I}+F_{J}}{2}-\mathcal{E}\right) \frac{\partial}{\partial \mathbf{c}_{I}}\left[\frac{S_{I J}}{\sqrt{\left\langle\Phi_{I} \mid \Phi_{I}\right\rangle\left\langle\Phi_{J} \mid \Phi_{J}\right\rangle}}\right] .
\end{aligned}
$$

In the notation we have developed, we can then write

$$
\begin{aligned}
\mathbf{b}_{I}= & -M_{b}\left[\left(\hat{V}_{I}+\hat{V}_{J}\right) / 2\right]+\left(\frac{F_{I}+F_{J}}{2}-\mathcal{E}\right) M_{b}[\hat{S}]+\frac{1}{2} \sum_{l} M_{b}\left[\hat{w}_{I, l}\right] \\
& -\frac{1}{2} \sum_{l} \frac{\partial V_{I, \ell}}{\partial \mathbf{c}_{I}}\left(N_{I, \ell} S_{I J}-\left\langle\Phi_{I}\left|\hat{w}_{I, \ell}\right| \Phi_{J}\right\rangle\right)
\end{aligned}
$$


where the $\partial V_{I, l} / \partial \mathbf{c}_{I}$ are determined from Eq. (55); $\mathbf{b}_{J}$ is determined similarly. A pair of GMRES calculations then yields $\mathbf{t}_{I}$ and $\mathbf{t}_{J}$, which are assembled into the final

$$
\frac{d W_{I J}}{d x}=-\tilde{M}_{x}\left[\left(\hat{V}_{I}+\hat{V}_{J}\right) / 2\right]+\left(\frac{F_{I}+F_{J}}{2}-\mathcal{E}\right) \tilde{M}_{x}[\hat{S}]-\tilde{L}_{x}\left(\mathbf{c}_{I}, \mathbf{t}_{I}\right)-\tilde{L}_{x}\left(\mathbf{c}_{J}, \mathbf{t}_{J}\right),
$$

noting that the $\mathbf{F}^{(x)}$ matrices which are used in computing the $\tilde{L}_{x}$ must include the contributions from $\hat{V}^{(x)}=\frac{\partial V}{\partial x} \hat{w}+V \hat{w}^{x}$ (where $\partial V / \partial x$ come from Eq. (49)).

This completes the construction of $W_{I J}^{x}$ and returns the gradient calculation of $\mathcal{E}^{x}$ to Eq. (5), recalling that $W_{I I}^{x}$ comes from Eq. (46). We note that the CI vector $C$ used in Eq. (5) is an eigenvector of the generalized eigenvector problem, in contrast to the coefficient vector produced by many CDFT-CI calculations, which is in the orthogonalized diabatic basis; a factor of $\mathbf{S}^{-1 / 2}$ allows interconversion.

\section{RESULTS}

We have implemented CDFT-CI gradients in a development version of Q-CHEM, and confirmed that our analytic gradients are correct by testing them against finite difference results. Since we do not have the benefit of the Hellmann-Feynman theorem for gradients, we expect that the CPU time for a CDFT-CI gradient evaluation should be comparable to that needed for a Hessian evaluation using regular DFT. As some indication of the qualitative similarity, we note that for the $\mathrm{OH}+\mathrm{C}_{2} \mathrm{H}_{6} \leftrightarrow \mathrm{H}_{2} \mathrm{O}+\mathrm{C}_{2} \mathrm{H}_{5}$ system presented below (with the 6-311++ $\mathrm{G}^{* *}$ basis, and 100 radial and 302 angular grid points), some timings are presented in Table I. The gradient evaluation is within roughly a factor of one and half of a DFT hessian evaluation, which is reasonable for our comparatively unoptimized code. We have endeavored to retain the $O\left(N^{3}\right)$ scaling behavior of DFT, albeit with a rather large prefactor. (Each GMRES iteration requires some number of $O\left(N^{3}\right)$ matrix manipulations, and it is not atypical for 20 GMRES iterations to be required for convergence.)

\section{A. Transition State Optimization}

The present work found it opportune to return to the set of reactions previously used to evaluate CDFT-CI[88], taken from the HTBH38/04 and NHTBH38 databases of Zhao et al.[94, 96] The newly implemented analytic gradients allow us to locate optimized transitionstate geometries at the CDFT-CI level of theory, to compare against the reference geometries 
which were optimized at a QCISD/MG3 level of theory.[94] Since the CI vector should be strongly spread over both configurations at the transition-state, these geometry optimizations represent a stringent test of the CDFT-CI coupling gradient computation - any inaccuracies in $W_{I J}^{x}$ would be highlighted in $\mathcal{E}^{x}$ by the delocalized CI vector. Furthermore, the change between the CDFT-CI energy calculated at the reference geometry and at the CDFT-CI-optimized geometry presents a measure of the quality of the CDFT-CI geometry; systems with small energy change are expected to have a converged CDFT-CI geometry close to the reference geometry. It also presents an opportunity to once again examine the overall quality of CDFT-CI for barrier heights. Unfortunately, the SG-1[97] quadrature grid used in Reference [88] is of insufficient quality to give reliable GMRES calculations, so we cannot reuse the data from that study directly. As such, the present calculations are performed using a $6-311++\mathrm{G}^{* *}$ basis set, as opposed to the $6-311+\mathrm{G}(3 \mathrm{df}, 2 \mathrm{p})$ basis set used in Reference [88], and the quality of the DFT integration grid is increased to a Lebedev grid with 100 radial points and 302 angular points (though the quality of the grid is less critical when a smaller basis set is used). The exchange-correlation functional used for CDFT-CI is B3LYP.[95] We deem it sufficient to present results using a single functional, given the overall robust performance with multiple functionals in the previous work.[88] Table II shows the results for the 32 reactions considered, with forward (and backward, when distinct) reaction barrier heights for CDFT-CI at the reference geometry, CDFT-CI at the optimized geom-

\begin{tabular}{ccc}
\hline \hline & BLYP & B3LYP \\
\hline$\partial V / \partial \mathbf{c}$ & 57 & 60 \\
$W^{x}$ & 475 & 474 \\
$S^{x}, F^{(x)}$ & 559 & 561 \\
$N^{x}$ & 1468 & 1651 \\
$V^{x}$ & 44 & 48 \\
$H^{x}-\mathcal{E} S^{x}$ & 739 & 1009 \\
\hline CDFT-CI gradient & 3342 & 3804 \\
DFT Hessian & 2272 & 2432 \\
\hline
\end{tabular}

TABLE I. Execution time for each term, in seconds of CPU time. 
etry, and the reference barrier heights. It also presents geometrical RMSD's of CDFT-CI and B3LYP compared against the reference transition state geometries; stock DFT geometries were located by using Gromacs[98]. There are some reactions where CDFT-CI and/or B3LYP were unable to determine a reaction transition state, either because GMRES did not converge or because there was no barrier predicted. In these cases, the reaction was excluded from the average RMSD computation for both the CDFT-CI and stock DFT averages.

\begin{tabular}{|c|c|c|c|c|c|}
\hline \multirow[b]{2}{*}{ Reaction } & \multicolumn{3}{|c|}{ Barrier heights } & \multicolumn{2}{|c|}{ Geom. RMSD } \\
\hline & $\begin{array}{l}\text { QCISD at } \\
\text { QCISD geom. }\end{array}$ & $\begin{array}{l}\text { CDFT-CI at } \\
\text { QCISD geom. }\end{array}$ & $\begin{array}{c}\text { CDFT-CI at } \\
\text { CDFT-CI geom. }\end{array}$ & CDFT-CI & B3LYP \\
\hline \multirow[t]{2}{*}{$1 \mathrm{H}+\mathrm{HCl} \leftrightarrow \mathrm{Cl}+\mathrm{H}_{2}$} & 5.7 & 4.71 & 5.66 & \multirow{2}{*}{0.042} & \multirow{2}{*}{0.060} \\
\hline & 8.7 & 11.09 & 12.05 & & \\
\hline \multirow[t]{2}{*}{$2 \mathrm{OH}+\mathrm{H}_{2} \leftrightarrow \mathrm{H}+\mathrm{H}_{2} \mathrm{O}$} & 5.1 & 7.86 & 6.35 & \multirow{2}{*}{0.010} & \multirow{2}{*}{0.032} \\
\hline & 21.2 & 19.66 & 18.15 & & \\
\hline \multirow[t]{2}{*}{$3 \mathrm{CH}_{3}+\mathrm{H}_{2} \leftrightarrow \mathrm{H}+\mathrm{CH}_{4}$} & 12.1 & 12.60 & 12.69 & \multirow{2}{*}{0.010} & \multirow{2}{*}{0.009} \\
\hline & 15.3 & 13.51 & 13.60 & & \\
\hline \multirow[t]{2}{*}{$4 \mathrm{OH}+\mathrm{CH}_{4} \leftrightarrow \mathrm{CH}_{3}+\mathrm{H}_{2} \mathrm{O}$} & 6.7 & 14.54 & - & \multirow{2}{*}{-} & \multirow{2}{*}{0.025} \\
\hline & 19.6 & 25.43 & - & & \\
\hline $5 \mathrm{H}+\mathrm{H}_{2} \leftrightarrow \mathrm{H}_{2}+\mathrm{H}$ & 9.6 & 7.64 & 7.64 & 0.003 & 0.002 \\
\hline \multirow[t]{2}{*}{$6 \mathrm{OH}+\mathrm{NH}_{3} \leftrightarrow \mathrm{H}_{2} \mathrm{O}+\mathrm{NH}_{2}$} & 3.2 & 2.80 & 2.89 & \multirow{2}{*}{0.105} & \multirow{2}{*}{0.210} \\
\hline & 12.7 & 11.47 & 11.56 & & \\
\hline \multirow[t]{2}{*}{$7 \mathrm{HCl}+\mathrm{CH}_{3} \leftrightarrow \mathrm{Cl}+\mathrm{CH}_{4}$} & 1.7 & 6.27 & 5.81 & \multirow{2}{*}{0.055} & \multirow{2}{*}{0.053} \\
\hline & 7.9 & 13.57 & 13.11 & & \\
\hline \multirow[t]{2}{*}{$8 \mathrm{OH}+\mathrm{C}_{2} \mathrm{H}_{6} \leftrightarrow \mathrm{H}_{2} \mathrm{O}+\mathrm{C}_{2} \mathrm{H}_{5}$} & 3.4 & 6.83 & - & \multirow{2}{*}{-} & \multirow{2}{*}{0.361} \\
\hline & 19.9 & 22.28 & - & & \\
\hline \multirow[t]{2}{*}{$9 \mathrm{~F}+\mathrm{H}_{2} \leftrightarrow \mathrm{H}+\mathrm{HF}$} & 1.8 & 1.72 & 1.32 & \multirow{2}{*}{0.051} & \multirow{2}{*}{-} \\
\hline & 33.4 & 29.64 & 29.24 & & \\
\hline \multirow[t]{2}{*}{$10 \mathrm{O}+\mathrm{CH}_{4} \leftrightarrow \mathrm{OH}+\mathrm{CH}_{3}$} & 13.7 & 23.72 & 17.93 & \multirow{2}{*}{0.075} & \multirow{2}{*}{0.007} \\
\hline & 8.1 & 19.81 & 14.02 & & \\
\hline \multirow[t]{2}{*}{$11 \mathrm{H}+\mathrm{PH}_{3} \leftrightarrow \mathrm{PH}_{2}+\mathrm{H}_{2}$} & 3.1 & 3.21 & 3.29 & \multirow{2}{*}{0.015} & \\
\hline & 23.2 & 27.63 & 27.70 & & 0.065 \\
\hline $12 \mathrm{H}+\mathrm{HO} \leftrightarrow \mathrm{H}_{2}+\mathrm{O}$ & 10.7 & 9.23 & 11.41 & & \\
\hline & 13.1 & 12.23 & 14.41 & 0.053 & 0.003 \\
\hline $13 \mathrm{H}+\mathrm{H}_{2} \mathrm{~S} \leftrightarrow \mathrm{H}_{2}+\mathrm{HS}$ & 3.5 & 5.20 & 4.78 & & \\
\hline & 17.3 & 22.56 & 22.14 & 0.032 & 0.045 \\
\hline $14 \mathrm{O}+\mathrm{HCl} \leftrightarrow \mathrm{OH}+\mathrm{Cl}$ & 9.8 & 5.10 & 5.86 & & \\
\hline & 10.4 & 8.49 & 9.25 & 0.028 & 0.044 \\
\hline
\end{tabular}




\begin{tabular}{|c|c|c|c|c|c|}
\hline \multirow[t]{2}{*}{$15 \mathrm{NH}_{2}+\mathrm{CH}_{3} \leftrightarrow \mathrm{CH}_{4}+\mathrm{NH}$} & 8.0 & 9.70 & 9.69 & \multirow{2}{*}{0.119} & \multirow{2}{*}{0.054} \\
\hline & 22.4 & 21.92 & 21.91 & & \\
\hline \multirow[t]{2}{*}{$16 \mathrm{NH}_{2}+\mathrm{C}_{2} \mathrm{H}_{5} \leftrightarrow \mathrm{C}_{2} \mathrm{H}_{6}+\mathrm{NH}$} & 7.5 & 11.68 & 11.10 & \multirow{2}{*}{0.37} & \multirow{2}{*}{0.034} \\
\hline & 18.3 & 19.34 & 18.77 & & \\
\hline \multirow[t]{2}{*}{$17 \mathrm{C}_{2} \mathrm{H}_{6}+\mathrm{NH}_{2} \leftrightarrow \mathrm{NH}_{3}+\mathrm{C}_{2} \mathrm{H}_{5}$} & 10.4 & 12.97 & 12.83 & \multirow{2}{*}{0.038} & \multirow{2}{*}{0.108} \\
\hline & 17.4 & 19.75 & 19.60 & & \\
\hline \multirow{2}{*}{$18 \mathrm{NH}_{2}+\mathrm{CH}_{4} \leftrightarrow \mathrm{CH}_{3}+\mathrm{NH}_{3}$} & 14.5 & 15.54 & 15.39 & \multirow{2}{*}{0.111} & \multirow{2}{*}{0.174} \\
\hline & 17.8 & 17.76 & 17.60 & & \\
\hline \multirow[t]{2}{*}{$19 \mathrm{H}+\mathrm{N}_{2} \mathrm{O} \leftrightarrow \mathrm{OH}+\mathrm{N}_{2}$} & 18.14 & 15.63 & 16.21 & \multirow{2}{*}{0.031} & \multirow{2}{*}{0.022} \\
\hline & 83.22 & 79.40 & 79.98 & & \\
\hline $20 \mathrm{H}+\mathrm{FH} \leftrightarrow \mathrm{FH}+\mathrm{H}$ & 42.18 & 37.29 & 37.12 & 0.007 & 0.004 \\
\hline $21 \mathrm{H}+\mathrm{ClH} \leftrightarrow \mathrm{HCl}+\mathrm{H}$ & 18.0 & 22.39 & 22.00 & 0.009 & 0.003 \\
\hline \multirow[t]{2}{*}{$22 \mathrm{H}+\mathrm{FCH}_{3} \leftrightarrow \mathrm{HF}+\mathrm{CH}_{3}$} & 30.38 & 26.03 & 25.93 & \multirow{2}{*}{0.013} & \multirow{2}{*}{0.009} \\
\hline & 57.02 & 53.61 & 53.51 & & \\
\hline \multirow{2}{*}{$23 \mathrm{H}+\mathrm{F}_{2} \leftrightarrow \mathrm{HF}+\mathrm{F}$} & 2.27 & -1.34 & -0.23 & \multirow{2}{*}{0.040} & \multirow{2}{*}{-} \\
\hline & 106.18 & 104.69 & 105.80 & & \\
\hline \multirow[t]{2}{*}{$24 \mathrm{CH}_{3}+\mathrm{FCl} \leftrightarrow \mathrm{CH}_{3} \mathrm{~F}+\mathrm{Cl}$} & 7.43 & 0.94 & 1.321 & \multirow{2}{*}{0.073} & \multirow{2}{*}{-} \\
\hline & 60.17 & 58.49 & 58.86 & & \\
\hline $25 \mathrm{~F}^{-}+\mathrm{CH}_{3} \mathrm{~F} \leftrightarrow \mathrm{FCH}_{3}+\mathrm{F}^{-}$ & -0.34 & 0.55 & 0.25 & 0.032 & 0.033 \\
\hline $26 \mathrm{~F}^{-} \cdots \mathrm{CH}_{3} \mathrm{~F} \leftrightarrow \mathrm{FCH}_{3} \cdots \mathrm{F}^{-}$ & 13.38 & 14.76 & 14.61 & & \\
\hline $27 \mathrm{Cl}^{-}+\mathrm{CH}_{3} \mathrm{Cl} \leftrightarrow \mathrm{ClCH}_{3}+\mathrm{Cl}^{-}$ & 3.10 & 1.56 & 1.43 & 0.026 & 0.044 \\
\hline $28 \mathrm{Cl}^{-} \cdots \mathrm{CH}_{3} \mathrm{Cl} \leftrightarrow \mathrm{ClCH}_{3} \cdots \mathrm{Cl}^{-}$ & 13.61 & 11.28 & 11.36 & & \\
\hline \multirow[t]{2}{*}{$29 \mathrm{~F}^{-}+\mathrm{CH}_{3} \mathrm{Cl} \leftrightarrow \mathrm{FCH}_{3}+\mathrm{Cl}^{-}$} & -12.54 & -13.28 & -13.45 & \multirow{2}{*}{0.024} & \multirow{2}{*}{0.014} \\
\hline & 20.11 & 20.94 & 20.77 & & \\
\hline \multirow[t]{2}{*}{$30 \mathrm{~F}^{-} \ldots \mathrm{CH}_{3} \mathrm{Cl} \leftrightarrow \mathrm{FCH}_{3} \cdots \mathrm{Cl}^{-}$} & 2.89 & 2.53 & 2.56 & & \\
\hline & 29.62 & 29.75 & 29.75 & & \\
\hline \multirow[t]{2}{*}{$31 \mathrm{OH}^{-}+\mathrm{CH}_{3} \mathrm{~F} \leftrightarrow \mathrm{HOCH}_{3}+\mathrm{F}^{-}$} & -2.78 & -3.23 & -3.24 & \multirow{2}{*}{0.116} & \multirow{2}{*}{0.116} \\
\hline & 17.33 & 18.65 & 18.64 & & \\
\hline $32 \mathrm{OH}^{-} \cdots \mathrm{CH}_{3} \mathrm{~F} \leftrightarrow \mathrm{HOCH}_{3} \cdots \mathrm{F}^{-}$ & 10.96 & 11.28 & 11.42 & & \\
\hline & 47.20 & 46.91 & 47.25 & & \\
\hline Average & & & & 0.043 & 0.048 \\
\hline
\end{tabular}


TABLE II: Reaction barrier heights from various methods in $\mathrm{kcal} / \mathrm{mol}$; forward and backward reaction barrier heights are shown when distinct. Reference heights using QCISD/MG3 taken from References [94] and [96], the CDFT-CI energy at the reference geometry, and the CDFT-CI energy at the optimized CDFT-CI geometry are shown. The CDFT-CI and stock DFT calculations used the $6-311++\mathrm{G}^{* *}$ basis set. For further comparison, also shown are geometrical RMSD's $(\AA)$ of CDFT-CI and B3LYP optimized geometries against the reference geometries. To avoid double-counting of transition states which are repeated in multiple reactions, RMSDs are not reported for reactions 26, 28, 30, and 32 (and are not included in the average). If a transition state failed to converge with either CDFT-CI or stock DFT, that reaction was excluded from the average RMSD.

Given that CDFT-CI with the reactant/product constrained states as its basis degenerates into ordinary DFT calculations on the reactant or product fragments at infinite separation, in some cases there is conflict between accurate forward and backward barrier heights, when DFT does not treat the reactant and product states equally well. Over the entire set of reactions (modulo those with no CDFT-CI transition state yet converged), CDFT-CI at the reference geometry has a mean error $(\mathrm{ME})$ of $0.56 \mathrm{kcal} / \mathrm{mol}$ and a mean absolute error of $2.63 \mathrm{kcal} / \mathrm{mol}$; the energies from optimized geometries give essentially the same results, with $\mathrm{ME}$ of $0.09 \mathrm{kcal} / \mathrm{mol}$ and mean absolute error $2.07 \mathrm{kcal} / \mathrm{mol}$. For comparison, the reference QCISD/MG3 results are only expected to be accurate within about one $\mathrm{kcal} / \mathrm{mol}$. Our previous work on these transition states used a different basis set and integration grid, but showed an improvement by approximately a factor of two going from the stock DFT energy at the reference geometry to the CDFT-CI energy at the reference geometry; for B3LYP the ME went from -5.0 to $1.2 \mathrm{kcal} / \mathrm{mol}$ and the MAE went from 5.1 to $2.5 \mathrm{kcal} / \mathrm{mol}$.[88] Splitting the reactions by class, average results are shown in Table III. There appear to be no substantial differences between the reference geometry results and the optimized geom- 


\begin{tabular}{crrrr}
\hline \hline & ME, initial & ME, optimized & MAE, initial & MAE, optimized \\
\hline Hydrogen transfer & 1.72 & 0.98 & 2.68 & 2.17 \\
Heavy atom transfer & -2.78 & -2.45 & 3.66 & 3.25 \\
Nucleophilic substitution & -0.07 & -0.10 & 0.88 & 0.84 \\
All reactions & 0.56 & 0.09 & 2.63 & 2.07 \\
\hline
\end{tabular}

TABLE III. Deviation of CDFT-CI barrier heights from the reference values. MEs and mean absolute errors (MAE) are given, broken down by the type of reaction, at the initial (reference) geometry, and at the final optimized geometry. All values in kcal/mol.

etry results at a per-category level, with the optimized geometries consistently performing slightly better. The geometrical RMSDs listed in Table II average to an ME of $0.043 \AA$ for the metric of geometrical RMSD (excluding the reactions where a transition state was not located by CDFT-CI and/or stock DFT). CDFT-CI slightly outperforms B3LYP (ME $0.048 \AA$ ) in getting the correct geometries, but there are some reactions where CDFT-CI and B3LYP can have a rather large RMSD from the reference. Some of these systems have rotational degrees of freedom which have minimal impact on the energy; these and other energetically weak distortions may explain some of the large RMSDs without indicating a poor description of the system.

Overall, CDFT-CI seems to produce very good reaction transition states, being essentially statistically indistinguishable from the reference geometries with respect to barrier heights, although the actual geometries can be slightly different. We should note that the results might be slightly better if symmetrized (e.g., spherically averaged) promolecule densities were used.

\section{B. Excited-state optimizations}

Gradients on the ground state allow for geometry optimization of critical points, both minima and saddle points (transition states). It is less common to have gradients of the excited state energy available, which enable optimization of minima on excited-state electronic PESs. Our CDFT-CI gradient implementation can produce forces on both the ground and excited states, treating them on an equal footing. As a simple example, we optimize the first singlet excited state of $\mathrm{H}_{2}$. This molecule has been exhaustively studied, and it is known that 
the first ${ }^{1} \Sigma_{g}^{+}$excited state has two minima, with the lower-energy minimum at a separation of $1.0 \AA$ and a second minimum at $2.3 \AA$. [99] The two minima correspond, qualitatively, to a $1 s \rightarrow 2 s$ Rydberg excitation and an linear combination of ionic states, respectively. Since CDFT is unable to describe valence excitations, it would be surprising if CDFT-CI could reproduce this double-minimum in the excited state. In fact, with the cc-pVDZ basis set and the B3LYP functional, using the standard four-state CDFT-CI active space for diatomics $\left(\mathrm{H}^{+} \mathrm{H}^{-}, \mathrm{H}^{-} \mathrm{H}^{+}, \mathrm{H}^{\uparrow} \mathrm{H}^{\downarrow}\right.$, and $\left.\mathrm{H}^{\downarrow} \mathrm{H}^{\uparrow}\right)$, we find only a single minimum at a separation of 2.235 $\AA$ (from an optimization starting at the ground-state equilibrium geometry). Throughout the optimization, the state in question is dominated by contributions from the ionic configurations, as expected given the unavailability of valence-excitation states. Nonetheless, the ionic minimum seems to be treated correctly, given that our modest basis set is not intended to yield quantitatively accurate results. It remains telling, though, that only one minimum is found - a reminder that we must always be conscious of the composition of the active space. If the active space does not include the proper states to describe a portion of the configuration space, then the CDFT-CI energy will be unreliable; this active space dependence must be kept in mind when applying CDFT-CI to new systems.

$\mathrm{H}_{2}$ is well studied in part because it is a very small test system, and it functions as a first test for new theoretical methods. However, the double-minimum in the excited state makes it less useful for assessing the validity of CDFT-CI given that we know CDFT-CI will fail for the valence excitations which comprise half of the double well. It is therefore useful to consider another simple molecule with well-known structure, but which has only a single minimum in the excited state. The diatomic $\mathrm{Li}_{2}$ meets these criteria; it also has more than two electrons, presenting a somewhat more stringent test on the applicability of theoretical methods. A similar four-state geometry optimization on the first singlet excited state of $\mathrm{Li}_{2}$ (also using cc-pVDZ and B3LYP) locates a geometry minimum (ungerade) at a separation of $3.187 \AA$. Furche and coworkers[29, 30] have compiled a benchmark suite of reference adiabatic excitation energies (including relaxation in the excited state), including excited-state geometries for for more than twenty molecules.[29] They give the dilithium ${ }^{1} \Sigma_{u}^{+}$minimum to be at $3.11 \AA$ (experiment[100]), with all the tabulated TD-DFT methods underpredicting the minimum except for TDHF. Given the proper active space, CDFT-CI successfully optimizes geometries for HOMO $\rightarrow$ LUMO excitations in these simple systems.

These diatomic systems do not present a compelling case for the necessity of analytic 
gradients with their single degree of freedom; moving to the ethylene cation adds more degrees of freedom while retaining a chemically simple system. It presents theoretical interest even in the ground state, in particular with the non-planar nature of the equilibrium

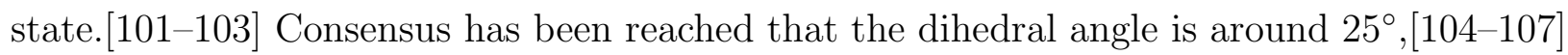
but it is difficult to confidently state a more precise value. The doublet nature of this molecule allows for only a four-state CDFT-CI active space to be used once again, splitting the molecule in half through the carbon-carbon bond, so that the two fragments are both $\mathrm{CH}_{2}$ units. Between those fragments, there are two possible splits for each of the charge and the spin; the product space gives four possible constraints: $\mathrm{CH}_{2}^{+} \mathrm{CH}_{2}^{\uparrow}, \mathrm{CH}_{2}^{+} \mathrm{CH}_{2}^{\downarrow}, \mathrm{CH}_{2}^{\uparrow} \mathrm{CH}_{2}^{+}$, and $\mathrm{CH}_{2}^{\downarrow} \mathrm{CH}_{2}^{+}$. A CDFT-CI ground-state optimization with that four-CDFT-state basis and again cc-pVDZ/B3LYP finds the dihedral angle at convergence to be $35.6^{\circ}$, even larger than the stock DFT state at $28.3^{\circ}$. However, this angle is known to be sensitive to the quality of the basis set,[107] so we do not necessarily seek quantitative accuracy. Of more interest to us at present is the behavior in the excited state; the minimum on the excited state is known to be at a conical intersection with the ground state, at a perpendicular geometry.[102] A CDFT-CI geometry optimization in the first excited state (starting from the equilibrium ground state structure) proceeds to a perpendicular geometry which is degenerate with the ground state. Unfortunately, Q-CHEM's geometry optimizer does not treat conical intersections, so there is little more that may be said about this system at present. We are currently working along this line and will present our results on conical intersection in a future article.

The general pattern of a four-state CDFT-CI with simple charge/spin-constrained states has been successful in these previous applications, so it is natural to apply it to another polyatomic molecule (which does not have its excited-state minimum at a conical intersection). Ethane $\left(\mathrm{C}_{2} \mathrm{H}_{6}\right)$ can be partitioned similarly to a diatomic (into two methyl groups) but has additional nuclear degrees of freedom, giving a more clear advantage to analytical gradients for geometry optimization. The four CDFT-CI states are now $\mathrm{CH}_{3}^{+} \mathrm{CH}_{3}^{-}, \mathrm{CH}_{3}^{-} \mathrm{CH}_{3}^{+}$, $\mathrm{CH}_{3}^{\uparrow} \mathrm{CH}_{3}^{\downarrow}$, and $\mathrm{CH}_{3}^{\downarrow} \mathrm{CH}_{3}^{\uparrow}$. At the ground-state equilibrium geometry, the $\mathrm{C}-\mathrm{C}$ distance is 1.53 $\AA$ for CDFT-CI with B3LYP/cc-pVDZ; the excited-state minimum for the ionic-like configuration has the carbons some $3.06 \AA$ apart from each other (Figure 2). This is similar to the diatomics previously studied, a little more than twice the ground-state separation, indicating a commonality amongst the ionic-like minima. Here, the methyl groups have both become essentially planar and are parallel to each other, though they retain the staggered rotational 
(a)

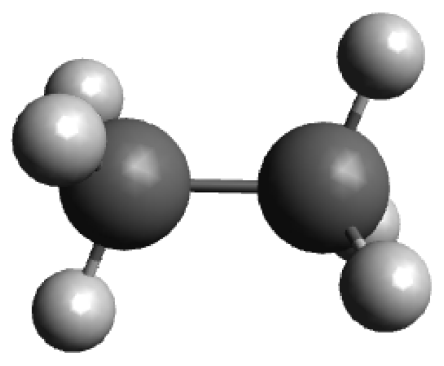

(b)
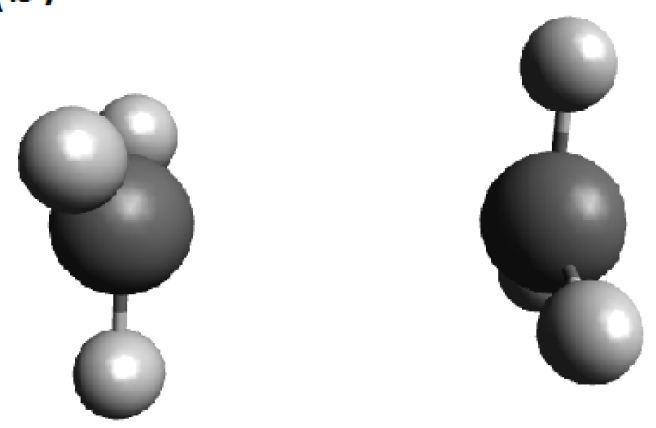

FIG. 2. Structures of ethane optimized by CDFT-CI: (a) ground state, (b) excited state.

conformation. The substantial geometry change is consistent with the mostly-continuous optical spectrum of ethane, given the minimal overlap with the ground state.[108] Again, CDFT-CI successfully locates the excited-state geometry of HOMO $\rightarrow$ LUMO excitations, given a sufficient active space.

As can be seen, with the CDFT-CI energy gradient for excited states, we are now able to study more about excited state chemistry by locating the geometries of conical intersections, and the energy minima of states with significant charge transfer and valence mixing, all of which the regular DFT cannot describe.

\section{CONCLUSIONS}

We have derived and implemented the equations necessary to obtain analytical gradients of the CDFT-CI energy. The resulting implementation has been used to validate previously investigated reaction barrier heights at self-consistently optimized transition-state geometries, which have good accuracy as compared against reference values computed by 
high-level theory. Gradients are available equally for the ground state and electronic excited states, allowing for optimization of excited state geometries. As a density-functional method, CDFT-CI has potential application to large systems, with gradients allowing for excited-state dynamics on organic photoelectronic systems at the donor/acceptor interface, even with QM/MM embedding. CDFT-CI gradients are not limited to just the gradient of the energy; the gradient of other one-electron properties such as the dipole moment and the density can be computed using the same machinery. There is also great potential in CDFTCI as an economical method for tracking the decay of optically excited systems, including decay to conical intersections. However, all this potential comes with a caveat, namely that the user must choose the active space for the calculation. Finding active spaces which remain valid over the entire area of the PES in question may prove to be challenging. Thus, it remains something of an open question what constitutes a "good" or "sufficient" active space for CDFT-CI calculations. Diatomics of low bond order seem well-understood, and the reactant/product split for the set of reaction transition states examined in this work produced good results, but no study has been made of whether increasing the active space would produce further improvement in transition states or elsewhere. Perhaps including configurations with charge-transfer character would shift the location of reaction transition states; the ability to optimize transition-state geometries allows any such effects to be studied, and the results used to give guidance for the selection of active spaces in general.

The availability of diabatic couplings and coupling gradients makes possible another investigation of key interest to chemists: studying the Condon approximation that the electronic coupling is relatively invariant to changes in nuclear position. Now that we have implemented the gradient of the coupling element between states, we can proceed to throw it away (set $S_{12}^{x}=H_{12}^{x}=0$ so that $\left.\mathcal{E}^{x} \approx C_{1}^{2} H_{11}^{x}+C_{2}^{2} H_{22}^{x}\right)$ and see how the omission changes the resulting nuclear dynamics. If the changes are small, then the Condon approximation can be safely applied for substantial computational speedup. Having the coupling derivative available allows the validity of the Condon approximation to be assessed on a system-by-system basis, giving greater confidence in the ensuing results.

Additionally, electronic excited states remain ever-tantalizing: to further assess CDFTCI's usability in this space, it would be fruitful to study simple photoisomerization systems. With only one bond changing, the difficulty of selecting a CDFT-CI active space is reduced, making isomerization studies feasible. Such studies would give insight into how to choose 
CDFT-CI active spaces for effective description of electronic excited states, helping to bring the DFT toolbox into scope for studying the photochemistry of more generic large molecules. Finally, with the CDFT-CI energy gradient now being efficiently available, an algorithm to locate minimum energy conical intersections should be developed, which will enable us to study photochemistry with CDFT-CI more thoroughly.

\section{ACKNOWLEDGEMENTS}

This work was supported by an NSF-CAREER Award (CHE-0547877). T.V. gratefully acknowledges a Packard Fellowship.

\section{Appendix A: Evaluating $V_{k}^{x}$}

We start off with knowledge that the constraints must be satisfied initially

$$
N_{k}=\left\langle\Phi\left|\hat{w}_{k}\right| \Phi\right\rangle
$$

A change in the constraint potential will manifest as a change in the Fock matrix, $\delta F$, which will induce a change in the wavefunction, $|\delta \Phi\rangle$. This change in the wavefunction will then contribute to the change in $N_{k}, \delta N_{k}$. Therefore,

$$
\begin{aligned}
\delta N_{k} & =\left\langle\Phi\left|\delta \hat{w}_{k}\right| \Phi\right\rangle+2\left\langle\Phi\left|\hat{w}_{k}\right| \delta \Phi\right\rangle \\
& =\left\langle\Phi\left|\delta \hat{w}_{k}\right| \Phi\right\rangle+2 \sum_{i}\left\langle\phi_{i}\left|\hat{w}_{k}\right| \delta \phi_{i}\right\rangle \\
& =\left\langle\Phi\left|\delta \hat{w}_{k}\right| \Phi\right\rangle+2 \sum_{i p}\left\langle\phi_{i}\left|\hat{w}_{k}\right| \phi_{p}\right\rangle\left\langle\phi_{p} \mid \delta \phi_{i}\right\rangle .
\end{aligned}
$$

The orbital overlaps $\left\langle\phi_{p} \mid \delta \phi_{i}\right\rangle$ are computed primarily from perturbation theory, taking care to include corrections from the nonorthogonal basis;

$$
\begin{aligned}
\left\langle\phi_{p} \mid \delta \phi_{i}\right\rangle & =\frac{\left\langle\phi_{p}\left|\delta \hat{F}-\epsilon_{i} \delta \hat{S}+\sum_{l} \delta \hat{V}_{l}\right| \phi_{i}\right\rangle}{\epsilon_{i}-\epsilon_{p}} \\
& =\frac{\left\langle\phi_{p}\left|\delta \hat{F}-\epsilon_{i} \delta \hat{S}+\sum_{l}\left\{\left(\delta V_{l}\right) \hat{w}_{l}+V_{l} \delta \hat{w}_{l}\right\}\right| \phi_{i}\right\rangle}{.} \epsilon_{i}-\epsilon_{p}
\end{aligned}
$$

This expression only holds for $i \neq p$; the contribution from $p=i$ can be determined from the normalization constraint on the orbitals;

$$
\left\langle\phi_{i} \mid \delta \phi_{i}\right\rangle=-\frac{1}{2}\left\langle\phi_{i}|\delta \hat{S}| \phi_{i}\right\rangle \text {. }
$$


There is no $\delta \epsilon_{i}$ contribution in Eqs. (A5) through (A7) because only variations of the orbitals which preserve normalization are produced.

Substituting Eqs. (A6) and (A7) into Eq. (A4) yields:

$$
\begin{aligned}
\delta N_{k}=\langle & \left.\left|\delta \hat{w}_{k}\right| \Phi\right\rangle \\
& +2 \sum_{i \neq p}\left\langle\phi_{i}\left|\hat{w}_{k}\right| \phi_{p}\right\rangle \frac{\left\langle\phi_{p}\left|\delta \hat{F}-\epsilon_{i} \delta \hat{S}+\sum_{l}\left\{\left(\delta V_{l}\right) \hat{w}_{l}+V_{l} \delta \hat{w}_{l}\right\}\right| \phi_{i}\right\rangle}{\epsilon_{i}-\epsilon_{p}} \\
& +2 \sum_{i}\left\langle\phi_{i}\left|\hat{w}_{k}\right| \phi_{i}\right\rangle\left\langle\phi_{i} \mid \delta \phi_{i}\right\rangle, \\
\delta N_{k}-\left\langle\Phi\left|\delta \hat{w}_{k}\right| \Phi\right\rangle=2 & \sum_{i \neq p}\left\langle\phi_{i}\left|\hat{w}_{k}\right| \phi_{p}\right\rangle \frac{\left\langle\phi_{p}\left|\sum_{l}\left(\delta V_{l}\right) \hat{w}_{l}\right| \phi_{i}\right\rangle}{\epsilon_{i}-\epsilon_{p}} \\
& +2 \sum_{i \neq p}\left\langle\phi_{i}\left|\hat{w}_{k}\right| \phi_{p}\right\rangle \frac{\left\langle\phi_{p}\left|\delta \hat{F}-\epsilon_{i} \delta \hat{S}+\sum_{l} V_{l} \delta \hat{w}_{l}\right| \phi_{i}\right\rangle}{\epsilon_{i}-\epsilon_{p}} \\
& -\sum_{i}\left\langle\phi_{i}\left|\hat{w}_{k}\right| \phi_{i}\right\rangle\left\langle\phi_{i}|\delta \hat{S}| \phi_{i}\right\rangle,
\end{aligned}
$$

Finally,

$$
\begin{gathered}
\delta N_{k}-\left\langle\Phi\left|\delta \hat{w}_{k}\right| \Phi\right\rangle+\sum_{i}\left\langle\phi_{i}\left|\hat{w}_{k}\right| \phi_{i}\right\rangle\left\langle\phi_{i}|\delta \hat{S}| \phi_{i}\right\rangle \\
-2 \sum_{i \neq p}\left\langle\phi_{i}\left|\hat{w}_{k}\right| \phi_{p}\right\rangle \frac{\left\langle\phi_{p}\left|\delta \hat{F}-\epsilon_{i} \delta \hat{S}+\sum_{l} V_{l} \delta \hat{w}_{l}\right| \phi_{i}\right\rangle}{\epsilon_{i}-\epsilon_{p}}=2 \sum_{l} \sum_{i \neq p}\left\langle\phi_{i}\left|\hat{w}_{k}\right| \phi_{p}\right\rangle \frac{\left\langle\phi_{p}\left|\hat{w}_{l}\right| \phi_{i}\right\rangle}{\epsilon_{i}-\epsilon_{p}}\left(\delta V_{l}\right), \\
b_{k}=A_{k}^{l}\left(\delta V_{l}\right),
\end{gathered}
$$

which is a system of linear equations corresponding to the various constraints being applied.

[1] I. S. K. Kerkines, I. D. Petsalakis, P. Argitis, and G. Theodorakopoulos, Phys. Chem. Chem. Phys. 13, 21273 (2011).

[2] D. Jacquemin, E. A. Perpète, G. Scalmani, M. J. Frisch, X. Assfeld, I. Ciofini, and C. Adamo, J. Chem. Phys. 125, 164324 (2006).

[3] M. Hartmann, J. Pittner, and V. Bonačić-Koutecký, J. Chem. Phys. 114, 2123 (2001).

[4] A. L. Kaledin and K. Morokuma, J. Chem. Phys. 113, 5750 (2000).

[5] U. Müller and G. Stock, J. Chem. Phys. 107, 6230 (1997). 
[6] D. Schemmel and M. Schütz, J. Chem. Phys. 133, 134307 (2010).

[7] A. L. Sobolewski and W. Domcke, J. Phys. Chem. A 111, 11725 (2007).

[8] J. D. Coe and T. J. Martínez, Mol. Phys. 106, 537 (2008).

[9] M. Etinski and C. M. Marian, Phys. Chem. Chem. Phys. 12, 4915 (2010).

[10] A. W. Lange and J. M. Herbert, J. Am. Chem. Soc. 131, 3913 (2009).

[11] H. R. Hudock and T. J. Martínez, ChemPhysChem 9, 2486 (2008).

[12] G. Groenhof, L. V. Schäfer, M. Boggio-Pasqua, M. Goette, H. Grubmüller, and M. J. Robb, J. Am. Chem. Soc. 129, 6812 (2007).

[13] W. J. Schreier, T. E. Schrader, F. O. Koller, P. Gilch, C. E. Crespo-Hernández, V. N. Swaminathan, T. Carell, W. Zinth, and B. Kohler, Science 315, 625 (2007).

[14] C. E. Crespo-Hernández, B. Cohen, and B. Kohler, Nature 436, 1141 (2005).

[15] T. Gustavsson, A. Bányász, E. Lazzarotto, D. Markovitsi, G. Scalmani, M. J. Frisch, V. Barone, and R. Improta, J. Am. Chem. Soc. 128, 607 (2006).

[16] J.-M. L. Pecourt, J. Peon, and B. Kohler, J. Am. Chem. Soc. 123, 10370 (2001).

[17] S. Matsika, J. Phys. Chem. A 108, 7584 (2004).

[18] H. R. Hudock, B. G. Levine, A. L. Thompson, and T. J. Martinez, AIP Conf. Proc. 963, 219 (2007).

[19] T. Kowalczyk, S. R. Yost, and T. Van Voorhis, J. Chem. Phys. 134, 054128 (2011).

[20] J. Cornil, D. Beljonne, J.-P. Calbert, and J.-L. Brédas, Adv. Mater. 13, 1053 (2001).

[21] S. Difley and T. Van Voorhis, J. Chem. Theory Comput. 7, 594 (2011).

[22] R. H. Friend, R. W. Gymer, A. B. Holmes, J. H. Burroughes, R. N. Marks, C. Taliani, D. D. C. Bradley, D. A. Dos Santos, J. L. Brédas, M. Lögdlund, and W. R. Salaneck, Nature 397, 121 (1999).

[23] J.-L. Brédas, J. E. Norton, J. Cornil, and V. Coropceanu, Acc. Chem. Res. 42, 1691 (2009).

[24] A. J. A. Aquino, M. Barbatti, and H. Lischka, ChemPhysChem 7, 2089 (2006).

[25] O. Lehtonen, D. Sundholm, and T. Vänskä, Phys. Chem. Chem. Phys. 10, 4535 (2008).

[26] L. Lapinski, M. J. Nowak, J. Nowacki, M. F. Rode, and A. L. Sobolewski, ChemPhysChem 10, 2290 (2009).

[27] C. V. Caillie and R. D. Amos, Chem. Phys. Lett. 308, 249 (1999).

[28] C. V. Caillie and R. D. Amos, Chem. Phys. Lett. 317, 159 (2000).

[29] F. Furche and R. Ahlrichs, J. Chem. Phys. 117, 7433 (2002). 
[30] R. Send, M. Kuhn, and F. Furche, J. Chem. Theory Comput. 7, 2376 (2011).

[31] M. Barbatti, G. Granucci, M. Persico, M. Ruckenbauer, M. Vazdar, M. Eckert-Maksić, and H. Lischka, J. Photochem. Photobiol. A 190, 228 (2007).

[32] J. Plötner and A. Dreuw, Chem. Phys. 347, 472 (2007).

[33] P. Wiggins, J. A. G. Williams, and D. J. Tozer, J. Chem. Phys. 131, 091101 (2009).

[34] A. Dreuw and M. Head-Gordon, Chem. Rev. 105, 4009 (2005).

[35] M. Wormit and A. Dreuw, J. Phys. Chem. B 110, 24200 (2006).

[36] J. B. Foresman, M. Head-Gordon, J. A. Pople, and M. J. Frisch, J. Phys. Chem. 96, 135 (1992).

[37] D. Maurice and M. Head-Gordon, Mol. Phys. 96, 1533 (1999).

[38] C. ik Song and Y. M. Rhee, J. Am. Chem. Soc. 133, 12040 (2011).

[39] R. Nithya, N. Santhanamoorthi, P. Kolandaivel, and K. Senthilkumar, J. Phys. Chem. A 115, 6594 (2011).

[40] W. J. Schreier, I. Pugliesi, F. O. Koller, T. E. Schrader, W. Zinth, and M. Braun, J. Phys. Chem. B 115, 3689 (2011).

[41] G. Bocchinfuso, C. Mazzuca, A. Palleschi, R. Pizzoferrato, and P. Tagliatesta, J. Phys. Chem. A 113, 14887 (2009).

[42] K. Andersson and B. O. Roos, Int. J. Quantum Chem. 45, 591 (1993).

[43] M. Merchán and B. O. Roos, Theor. Chim. Acta 92, 227 (1995).

[44] A. Rauk, D. Yu, P. Borowski, and B. Roos, Chem. Phys. 197, 73 (1995).

[45] C. S. Page and M. Olivucci, J. Comput. Chem. 24, 289 (2003).

[46] S. Olsen, K. Lamonthe, and T. J. Martinez, J. Am. Chem. Soc. 132, 1192 (2010).

[47] X. Yu, S. Yamazaki, and T. Taketsugu, J. Chem. Theory Comput. 7, 1006 (2011).

[48] P. B. Coto, A. Strambi, and M. Olivucci, Chem. Phys. 347, 483 (2008).

[49] P. Celani and H.-J. Werner, J. Chem. Phys. 119, 5044 (2003).

[50] J. F. Stanton, J. Chem. Phys. 99, 8840 (1993).

[51] J. F. Stanton and J. Gauss, J. Chem. Phys. 100, 4695 (1994).

[52] J. F. Stanton and J. Gauss, Theor. Chim. Acta 91, 267 (1995).

[53] L. A. Burns, D. Murdock, and P. H. Vaccaro, J. Chem. Phys. 130, 144304 (2009).

[54] D. Gerbig, H. P. Reisenauer, C.-H. Wu, D. Ley, W. D. Allen, and P. R. Schreiner, J. Am. Chem. Soc. 132, 7273 (2010). 
[55] O. Christiansen, J. F. Stanton, and J. Gauss, J. Chem. Phys. 108, 3987 (1998).

[56] A. Köhn and C. Hättig, J. Chem. Phys. 119, 5021 (2003).

[57] R. Brause, M. Santa, M. Schmitt, and K. Kleinermanns, ChemPhysChem 8, 1394 (2007).

[58] Y. Zhang, G. Burdzinski, J. Kubicki, S. Vyas, C. M. Hadad, M. Sliwa, O. Poizat, G. Buntinx, and M. S. Platz, J. Am. Chem. Soc. 131, 13784 (2009).

[59] E. V. Gromov, I. Burghardt, H. Köppel, and L. S. Cederbaum, J. Phys. Chem. A 115, 9237 (2011)

[60] S. Smolarek, A. Vdovin, A. Rijs, C. A. van Walree, M. Z. Zgierski, and W. J. Buma, J. Phys. Chem. A 115, 9399 (2011).

[61] M. F. Rode and A. L. Sobolewski, J. Phys. Chem. A 114, 11879 (2010).

[62] H.-J. Werner and E.-A. Reinsch, J. Chem. Phys. 76, 3144 (1982).

[63] R. Shepard, I. Shavitt, R. M. Pitzer, D. C. Comeau, M. Pepper, H. Lischka, P. G. Szalay, R. Ahlrichs, F. B. Brown, and J.-G. Zhao, Int. J. Quantum Chem. 34, 149 (1988).

[64] H. Lischka, R. Shepard, R. M. Pitzer, I. Shavitt, M. Dallos, T. Müller, P. G. Szalay, M. Seth, G. S. Kedziora, S. Yabushita, and Z. Zhang, Phys. Chem. Chem. Phys. 3, 664 (2001).

[65] H. Lischka, M. Dallos, and R. Shepard, Mol. Phys. 100, 1647 (2002).

[66] B. G. Levine, C. Ko, J. Quenneville, and T. J. Martínez, Mol. Phys. 104, 1039 (2006).

[67] B. Kaduk and T. Van Voorhis, J. Chem. Phys. 133, 061102 (2010).

[68] K. Giesbertz and E. Baerends, Chem. Phys. Lett. 461, 338 (2008).

[69] A. Dreuw and M. Head-Gordon, J. Am. Chem. Soc. 126, 4007 (2004).

[70] A. Dreuw, J. L. Weisman, and M. Head-Gordon, J. Chem. Phys. 119, 2943 (2003).

[71] M. J. G. Peach, P. Benfield, T. Helgaker, and D. J. Tozer, J. Chem. Phys. 128, 044118 (2008).

[72] S. Arulmozhiraja and M. L. Coote, J. Chem. Theory Comput. 8, 575 (2012).

[73] A. V. Kityk, J. Phys. Chem. A 116, 3048 (2012).

[74] R. M. Richard and J. M. Herbert, J. Chem. Theory Comput. 7, 1296 (2011).

[75] G. Sini, J. S. Sears, and J.-L. Brédas, J. Chem. Theory Comput. 7, 602 (2011).

[76] P. Romaniello, D. Sangalli, J. A Berger, F. Sottile, L. G. Molinari, L. Reining, and G. Onida, J. Chem. Phys. 130, 044108 (2009).

[77] D. Hofmann, T. Körzdörfer, and S. Kümmel, Phys. Rev. Lett. 108, 146401 (2012).

[78] N. Kuritz, T. Stein, R. Baer, and L. Kronik, J. Chem. Theory Comput. 7, 2408 (2011). 
[79] P. Elliott, S. Goldson, C. Canahui, and N. T. Maitra, Chem. Phys. 391, 110 (2011).

[80] M. Filatov and S. Shaik, Chem. Phys. Lett. 304, 429 (1999).

[81] I. Frank, J. Hutter, D. Marx, and M. Parrinell, J. Chem. Phys. 108, 4060 (1998).

[82] I. Okazaki, F. Sato, T. Yoshihiro, T. Ueno, and H. Kashiwagi, J. Mol. Struct. Theochem 451, 109 (1998).

[83] T. Kowalczyk, T. Tsuchimochi, P.-T. Chen, L. Top, and T. V. Voorhis, J. Chem. Phys. 138, 164101 (2013).

[84] Q. Wu and T. Van Voorhis, Phys. Rev. A 72, 024502 (2005).

[85] Q. Wu and T. Van Voorhis, J. Chem. Theory Comput. 2, 765 (2006).

[86] Q. Wu and T. Van Voorhis, J. Phys. Chem. A 110, 9212 (2006).

[87] Q. Wu, C.-L. Cheng, and T. Van Voorhis, J. Chem. Phys. 127, 164119 (2007).

[88] Q. Wu, B. Kaduk, and T. Van Voorhis, J. Chem. Phys. 130, 034109 (2009).

[89] R. McWeeny, Rev. Mod. Phys. 32, 335 (1960).

[90] This definition does give preference to $\mathbf{c}_{I}$ over $\mathbf{c}_{J}$, but this is immaterial given that the structure of $M$ is symmetric with respect to the two states.

[91] L. N. Trefethen and D. B. III, Numerical Linear Algebra, Society for Industrial and Applied Mathematics, Philadelphia, 1997.

[92] Y. Saad and M. H. Schultz, SIAM J. Sci and Stat. Comput. 7, 856 (1986).

[93] B. G. Johnson, P. M. W. Gill, and J. A. Pople, J. Chem. Phys. 98, 5612 (1993).

[94] Y. Zhao, N. González-García, and D. G. Truhlar, J. Phys. Chem. A 109, 2012 (2005).

[95] A. Becke, J. Chem. Phys. 98, 5648 (1993).

[96] Y. Zhao, B. J. Lynch, and D. G. Truhlar, Phys. Chem. Chem. Phys. 7, 43 (2005).

[97] P. M. W. Gill, B. G. Johnson, and J. A. Pople, Chem. Phys. Lett. 209, 506 (1993).

[98] B. Hess, C. Kutzner, D. van der Spoel, and E. Lindahl, J. Chem. Theory Comput. 4, 435 (2008).

[99] E. R. Davidson, J. Chem. Phys. 35, 1189 (1961).

[100] K.-P. Huber and G. Herzberg, Molecular Spectra and Molecular Structure. IV. constants of diatomic molecules, Van Nostrand Reinhold Co., New York, 1979.

[101] C. Sannen, G. Raşeev, C. Galloy, G. Fauville, and J. C. Lorquet, J. Chem. Phys. 74, 2402 (1981).

[102] H. Köppel, L. S. Cederbaum, and W. Domcke, J. Chem. Phys. 77, 2014 (1982). 
[103] K. Takeshita, J. Chem. Phys. 95, 1838 (1991).

[104] H. Köppel, W. Domcke, L. S. Cederbaum, and W. von Niessen, J. Chem. Phys. 69, 4252 (1978).

[105] P. M. Dehmer and J. L. Dehmer, J. Chem. Phys. 70, 4574 (1979).

[106] J. E. Pollard, D. J. Trevor, J. E. Reutt, Y. T. Lee, and D. A. Shirley, J. Chem. Phys. 81, $5302(1984)$.

[107] N. C. Handy, R. H. Nobes, and H.-J. Werner, Chem. Phys. Lett. 110, 459 (1984).

[108] G. Herzberg, Molecular Spectra and Molecular Structure. III. Electronic spectra and electronic structure of polyatomic molecules, Van Nostrand Co., Princeton, NJ, 1967. 


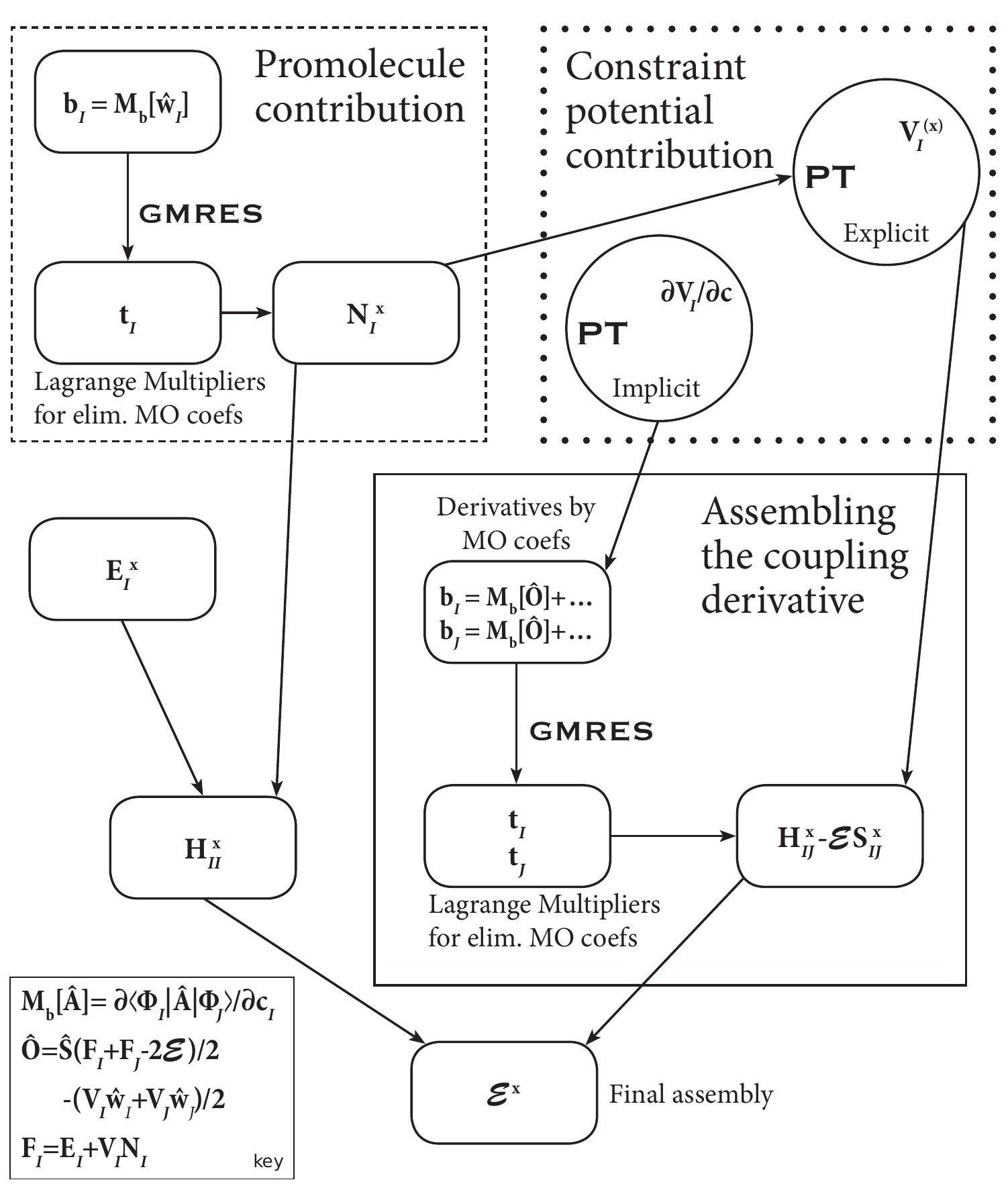


(a)

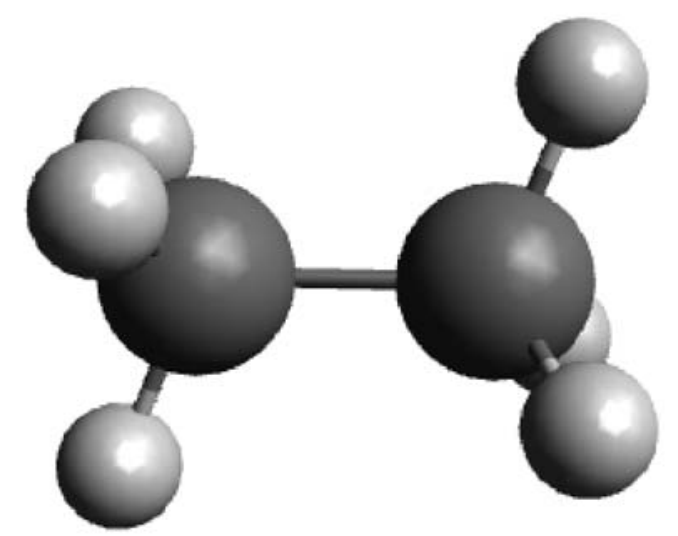

(b)
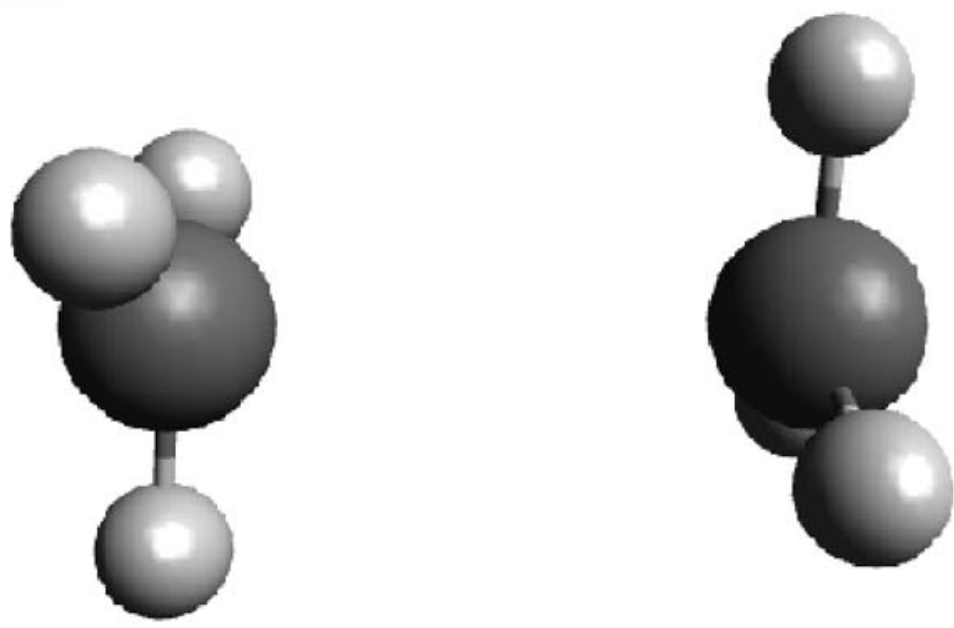